\title{
QUALITÉ CHIMIQUE ET BIOLOGIQUE DU BASSIN DE LA SEMOIS (PARTIE BELGE).
}

\section{LECLERCQ (1), F. ROSILLON (2) et P. VANDER BORGHT (2),} avec la collaboration de A. LONCIN (2) et M. EL MOSSAOUI (2).

(1) Université de Liège, Station scientifique des Hautes-Fagnes, 137 rue de Botrange, B-4950 Robertville, Belgique.

(2) Fondation universitaire luxembourgeoise, 185 avenue de Longwy, B-6700 Arlon, Belgique.

\section{RÉSUMÉ}

Les eaux du bassin de la Semois belge ont été étudiées, en 25 stations, sous différents aspects complémentaires : chimie, diatomées et invertébrés benthiques. Les méthodes utilisées sont l'indice de pollution organique (IPO, LECLERCQ et VANDEVENNE, 1987), un indice diatomique (ID, LECLERCQ, non publié) et l'indice biologique global normalisé (IBGN, AFNOR, 1992). Grâce aux analyses chimiques et diatomiques, nous décrivons différents types d'eaux naturelles, à minéralisation croissante, et différents niveaux de pollution et d'eutrophication. Nous présentons une carte de qualité détaillée, à l'usage des gestionnaires. Les diatomées apparaissent comme les bioindicateurs les plus fiables pour ces paramètres. Les invertébrés sont moins intéressants à utiliser. lis sont pratiquement insensibles au type de minéralisation de l'eau (tout au moins au niveau de la famille, qui est le seul niveau d'identification possible en routine), mais ces organismes peuvent être plus sensibles au facteur substrat qu'à la pollution organique, conduisant alors à des estimations erronées.

Mots-clés : Semois, chimie de l'eau, diatomées, invertébrés, indices biologiques.

\section{CHEMICAL AND BIOLOGICAL QUALITY OF THE BELGIAN SEMOIS BASIN.}

\section{SUMMARY}

The fresh water of the Belgian Semois catchment area was studied at 25 stations from the point of view of several complementary aspects : chemical composition, diatoms and benthic invertebrates. The methods used were the organic pollution index (IPO, LECLERCQ and VANDEVENNE, 1987), a diatom index (ID, LECLERCQ, unpublished) and the global normalized biological index (IBGN, AFNOR, 1992). The chemical and diatom analyses enabled us to describe several kinds of natural water subject to increasing mineralization and presenting various levels of pollution and eutrophication. We present a detailed quality map for the basin managers. Diatoms seem to be the most reliable bioindicators for these parameters. Invertebrates are less interesting, as they are practically insensitive to water mineralization (at least, at the family level which is the only possible identification level in routine monitoring) ; moreover, invertebrates may be more sensitive to the substrate than to organic pollution, which can lead to erroneous quality evaluations.

Key-words : Semois, water chemistry, diatoms, invertebrates, biological index. 


\section{INTRODUCTION}

En vue d'établir la qualité biologique des eaux du bassin de la Semois, nous avons procédé, du 27 au 29 juin 1995, à des prélèvements d'eau, d'algues et de macro-invertébrés benthiques en 12 stations sur la Semois et 13 stations sur les principaux affluents. Nous résumons ici une description détaillée de ce bassin par VANDER BORGHT et ROSILLON (1994).

La Semois prend sa source à Arlon (Belgique) et rejoint la Meuse à Monthermé (France), après un parcours de $210 \mathrm{~km}$ (figure 1). La partie supérieure, jusqu'aux environs de Florenville, se trouve sur substrat calcaire (Jurassique) puis on passe sur les terrains siliceux, essentiellement du Dévonien inférieur. La majorité des affluents sont en rive droite et viennent également de ces terrains siliceux. Le bassin versant s'étend sur $1329 \mathrm{~km}^{2}$.

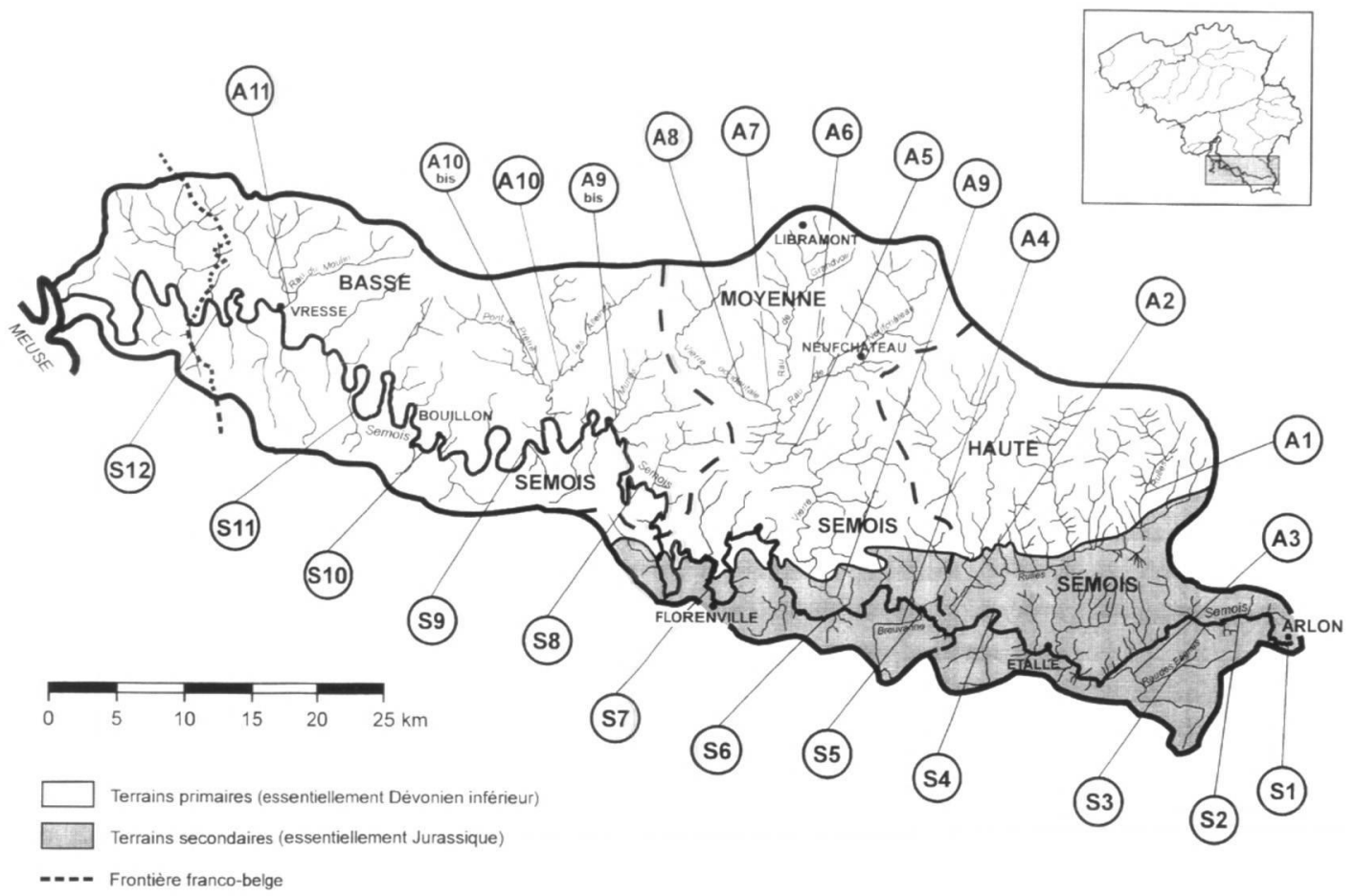

Figure 1 : Carte de situation de la Semois et des points de prélèvement.

Figure 1 : Map of the Semois basin and of the sampling stations.

Le bassin de la Semois comprend trois grandes divisions :

- le cours supérieur ou haute Semois, d'Arlon à Tintigny, soit $389 \mathrm{~km}^{2}$, avec un affluent important : la Rulles;

- le cours moyen ou moyenne Semois, jusqu'en aval de Sainte-Cécile, soit $405 \mathrm{~km}^{2}$, avec un affluent important : la Vierre ;

- le cours inférieur ou basse Semois, jusqu'à la confluence avec la Meuse, soit 525 km², avec trois affluents principaux : les Alleines, le ruisseau du Moulin et le Saint-Jean.

Le bassin de la Semois compte près de 75000 habitants, issus de 19 communes. C'est une région essentiellement rurale, sauf la ville d'Arlon située à la source de la Semois, dont 18000 habitants vivent dans le bassin.

La forêt occupe plus de $50 \%$ du territoire. Elle est particulièrement étendue dans les communes ardennaises qui connaissent un phénomène de reboisement, surtout en résineux, aux dépens de la surface agricole. L'agriculture, surtout orientée vers le pâturage, est cependant bien représentée : 95000 bovins sont élevés dans les 12 principales communes du bassin. Outre 
ces deux secteurs, la principale activité économique est le tourisme. 65 terrains de camping, totalisant 7000 emplacements, sont implantés en bordure de la Semois et des principaux affluents.

En dehors de l'activité agricole, la pollution en eaux usées du bassin est estimée à 120000 équivalents-habitant dont $60 \%$ sont épurés dans les 18 stations collectives, les principales étant situées à Arlon, Bertrix, Bouillon, Neufchâteau, Herbeumont. Mais, des réseaux d'assainissement parfois déficients d'une part, une topographie complexe et un habitat dispersé d'autre part, font que certains rejets d'eaux usées se déversent directement dans les cours d'eau. Ainsi, la ville d'Arlon a connu un développement récent de nouvelles activités industrielles et artisanales, accompagné de nouveaux rejets qui ne transitent pas encore par la station d'épuration. De nombreux villages déversent encore leurs eaux usées directement dans le réseau hydrographique.

A cette pollution due à la population autochtone s'ajoute, en période estivale, la charge générée par le secteur du tourisme. Les terrains de camping, dont peu sont équipés de dispositifs d'épuration, participent à la dégradation de la qualité des eaux en période d'étiage, notamment sur le plan bactériologique.

La présente étude s'intègre dans le cadre d'un “ Contrat de rivière " ( $\left.{ }^{*}\right)$, dont le secrétariat a été confié à la Fondation universitaire luxembourgeoise (VANDER BORGHT et ROSILLON, 1994), et vise à dresser une carte précise de la qualité des eaux du bassin.

Quelques données chimiques et diatomiques existent dans la littérature (PIERRE, 1977 ; DESCY, 1978 ; DESCY et al., 1982 ; VANDER BORGHT et SKA, 1989). Déjà anciennes et hétéroclites, comparativement à l'évolution récente de la situation (installation d'une station d'épuration, puis d'une importante zone industrielle dont les eaux usées ne transitent pas par cette station), il était urgent de les actualiser. II est donc de peu d'intérêt de faire ici une comparaison avec ces données anciennes si ce n'est pour compléter l'inventaire floristique des diatomées, ce qui șera fait dans une publication ultérieure.

II existe un grand nombre de méthodes d'estimation de la qualité des eaux courantes, basées sur la chimie et sur les organismes vivants. Le but n'est pas d'en faire ici un inventaire: nous renvoyons, pour cela, à d'autres publications ou rapports (AGENCE DE L'EAU, 1993 ; DE PAUW et VANHOOREN, 1983 ; LECLERCQ, 1991 ; ROUND, 1993).

Pour dresser un état de qualité complet et fiable, nous utilisons conjointement 4 méthodes s'adressant à des niveaux différents de l'écosystème : chimie de l'eau, diatomées et macroinvertébrés benthiques.

\section{MATÉRIEL ET MÉTHODES}

\subsection{Analyses chimiques}

Les paramètres ont été mesurés par les méthodes classiques : température, $\mathrm{pH}$, conductivité et oxygène dissous par sonde sur le terrain, l'alcalinité par titrimétrie, les sulfates par néphélométrie, les chlorures, nitrates, ammonium, nitrites et orthophosphates par colorimétrie (chaîne d'analyse automatique Technicon), le calcium, -magnésium, sodium et potassium par émission atomique à plasma (ICP).

\subsubsection{Diagrammes ioniques}

Ils sont tracés selon la méthode de KUFFERATH (1951).

(") Le contrat de rivière Semois résulte d'une convention entre le Ministère de l'Environnement de la Région wallonne et les 12 principales communes du bassin (Arlon, Bertrix, Bouillon, Chiny, Etalle, Florenville, Habay, Herbeumont, Leglise, Neufchâteau, Tintigny et Vresse). 
Ils donnent une image concrète de la minéralisation naturelle d'une eau et nécessitent l'analyse des éléments suivants : calcium, magnésium, sodium et potassium pour les cations ; sulfates, chlorures et carbonates-bicarbonates pour les anions. Les valeurs sont exprimées en milliéquivalents/l.

Cette minéralisation est en étroite relation avec la nature des roches du bassin versant et du lit (SYMOENS, 1957 ; LECLERCQ, 1988). D'une façon simplifiée, SYMOENS (1957) distingue, pour le sud de la Belgique, 4 types d'eau dont le nom fait référence à la région concernée : type fagnard dans les Hautes-Fagnes (eaux dystrophes très acides), type ardennais en Ardenne (eaux oligotrophes), type famennien en Famenne (type mésotrophe) et type condrusien dans le Condroz (type calcaire eutrophe).

Ces analyses sont importantes car, en cas de pollution, il importe de connaître le type chimique potentiel pour établir valablement le plan de gestion des eaux. Elles permettent aussi de comprendre la composition de la flore algale très sensible à ces paramètres, surtout au calcium et aux bicarbonates.

Généralement, dans le sud de la Belgique, les rivières coulent en amont sur les roches les plus pauvres en éléments échangeables, puis en aval sur des roches de plus en plus riches : on assiste alors à l'eutrophisation des eaux, définie comme étant l'enrichissement naturel en minéraux (calcium, magnésium, ...). Le diagramme ionique rend bien compte de ce processus.

\subsubsection{Indice de pollution organique et eutrophication}

Nous utilisons ici l'Indice de Pollution Organique (IPO, LECLERCQ et VANDEVENNE, 1987) calculé à partir des mesures d'ammonium, nitrites et orthophosphates, la $\mathrm{DBO}_{5}$ n'ayant pas été mesurée dans cette étude.

Les teneurs sont réparties en 5 classes, dont les limites ont été déterminées à partir des modifications observées dans les peuplements d'algues diatomées très sensibles à ces paramètres, et ce, par référence aux milieux naturels (près de 1000 prélèvements analysés dans le sud de la Beigique). Ces classes ont donc une signification écologique bien précise. Par exemple, la première classe des orthophosphates est inférieure à $15 \mu \mathrm{g}-\mathrm{P} / \mathrm{l}$, valeur a priori très faible, mais au-delà de laquelle les risques de prolifération algale commencent et des modifications apparaissent déjà dans les peuplements de diatomées.

Les valeurs indicielles sont réparties en 5 classes de pollution, avec un code de couleur (tableau 1).

D'autres méthodes font souvent intervenir la DCO, l'oxygène, les nitrates (BECKERS et STEEGMANS, 1979 ; PRATI et al., 1971 ; VERNIERS et MICHA, 1982). Ces paramètres sont ambigus. En effet, on connaît des types d'eau naturellement riches en acides humiques, qui présentent une DCO élevée. Par ailleurs, on constate que des eaux très polluées peuvent être riches en oxygène pour autant qu'elles soient torrentueuses. Enfin, les nitrates ne caractérisent pas la pollution organique, mais en sont la résuitante après minéralisation.

Tableau I : Classes de pollution pour les trois indices (IPO, ID, IBGN) et code des couleurs.

Table I : Categories of pollution for the three used indexes (IPO, ID, IBGN) and their colours.

\begin{tabular}{|l|c|c|c|c|}
\hline & IPO & ID & IBGN & code couleur \\
\hline pollution nulle & $5,0-4,6$ & $5,0-4,3$ & $20-17$ & bleu \\
pollution faible & $4,5-4,0$ & $4,2-3,6$ & $16-13$ & vert \\
pollution modérée & $3,9-3,0$ & $3,5-3,0$ & $12-9$ & jaune \\
pollution forte & $2,9-2,0$ & $2,9-2,3$ & $8-5$ & orange \\
pollution très forte & $1,9-1,0$ & $2,2-1,0$ & $4-1$ & rouge \\
\hline
\end{tabular}


Précisément, lorsque les matières organiques sont minéralisées, la rivière passe par un stade enrichi en orthophosphates et nitrates qui favorisent la prolifération de la biomasse végétale. Il s'agit aiors d'une eutrophication, terme clairement défini par.DUVIGNEAUD (1984, p. 248), à distinguer de l'eutrophisation, enrichissement naturel de l'amont vers l'aval en calcium, magnésium et bicarbonates.

Pour souligner le caractère anormal de l'eutrophication, on utilise aussi le terme " hypertrophisation " pour les milieux lentiques.

Au niveau végétal, l'eutrophication n'entraîne pas du tout les mêmes modifications que l'eutrophisation naturelle. Dans le premier cas, il y a surproduction végétale, et des espèces de diatomées affectionnant les nitrates et les orthophosphates se développent. Dans le second cas, des espèces sensibles, de plus en plus alcaliphiles, se succèdent.

Au niveau des invertébrés et des poissons, ces niveaux d'orthophosphates ne sont pas nocifs en eux-mêmes, mais c'est la prolifération de végétaux qui entraîne des mortalités.

Il n'existe pas encore d'indice satisfaisant pour quantifier l'eutrophication des eaux courantes. Nous la détecterons ici par la sursaturation diurne en oxygène, allant de pair avec un excès de végétaux aquatiques.

\subsection{Indices biologiques}

L'intérêt d'utiliser des bioindicateurs de la qualité de l'eau, en plus de l'analyse chimique, a été démontré depuis longtemps (KOLKWITZ et MARSSON, 1908).

On sait ainsi que la composition chimique d'une eau polluée, généralement estimée à partir d'un seul prélèvement instantané, est malheureusement influencée par les irrégularités des rejets, mais l'analyse chimique reste le seul moyen d'identifier la nature et les concentrations des polluants ; en revanche, les bioindicateurs, grâce à leur capacité d'intégration des variations des facteurs du milieu et à la bonne connaissance actuelle des exigences des différentes espèces, permettent de déterminer de façon très fiable, à partir d'un prélèvement également instantanë :

- le type d'eau naturel (ou le type apparent en cas de pollution par enrichissement artificiel de l'eau en sels minéraux) : pour cela, on recherche notamment les espèces acidophiles, neutrophiles oligotrophes et mésotrophes, alcaliphiles ;

- le niveau de pollution organique et en plus, pour les diatomées, le niveau de salinité naturelle ou induite (sodium, chlorures).

Nous utilisons ici les diatomées benthiques comme représentants de la phytocénose, et la macrofaune benthique pour la zoocénose.

\subsubsection{Les diatomées benthiques}

Les diatomées, producteurs primaires, dépendent directement des nutriments disponibles et sont particulièrement sensibles aux formes de l'azote et du phosphore, fréquentes dans les pollutions domestiques. Lorsque la teneur en ces éléments augmente dans l'eau, la mosaïque d'espèces se modifie rapidement : les espèces sensibles régressent au profit des espèces résistantes.

L'échantillonnage se fait par brossage des faces supérieures des pierres immergées en zone lotique, sur une surface d'environ $400 \mathrm{~cm}^{2}$. Danis beaucoup de cas, un seul prélèvement annuel peut suffire, mais cette fréquence eșt à moduler en fonction des circonstances (activité touristique saisonnière, par exemple). La préparation se fait par les méthodes classiques (nettoyage des frustules à l'acide nitrique à chaud, montage dans le Naphrax). On procède au comptage de 500 frustules et on complète la liste floristique par un balayage complémentaire, les taxons supplémentaires ainsi répertoriés comptant alors pour $1 / 2$ individu, soit $0,1 \%$ d'abondance dans le relevé. 
La systématique suivie est, à quelques exceptions près (voir chapitre 3.2.1.1.), celle de KRAMMER et LANGE-BERTALOT (1986-1991) et de KRAMMER (1992). On peut identifier toutes les espèces de l'échantillon, chacune ayant ses propres exigences écologiques : on en tire ainsi un maximum d'informations sur la qualité de l'eau.

L'indice que nous avons développé (LECLERCQ et MAQUET, 1987) est basé sur l'indice saprobique de SLADECEK (1973). Cette méthode attribue à chaque taxon spécifique ou infraspécifique une valence saprobique (vs) et une valeur indicatrice (vi) qui décrivent son auto-écologie ; une combinaison mathématique associe ces valeurs et l'abondance relative des taxons $(\mathrm{A})$ :

$$
\text { indice diatomique }=\frac{\Sigma v s_{X} \cdot v i_{X} \cdot A_{X}}{\Sigma v i_{X} \cdot A_{X}} \quad(x=1 \text { à } n, n \text { étant le nombre de taxons }) .
$$

Nous avons recalculé les vs et les vi, à partir des indications auto-écologiques issues de nos propres observations en Belgique et de nombreuses références bibliographiques pour les zones tempérées, et ce, pour plus de 400 taxons. Notre indice peut ainsi, en principe, être appliqué dans l'ensemble de ces régions. La publication de ces valeurs est envisagée.

L'indice diatomique varie de 5 à 1 en continu et est réparti en 5 classes de pollution croissante (tableau I).

\subsubsection{Les macro-invertébrés benthiques}

Dans ce cas, on prélève, sur le fond du cours d'eau, tous les invertébrés aquatiques macroscopiques appartenant à des groupes systématiques très différents (insectes adultes ou larves, vers, mollusques, ...). Ces organismes sont des consommateurs de premier ordre (herbivores) et de deuxième ou troisième ordre (carnivores).

La méthode de SLADECEK (1973) permet, comme pour les diatomées, de calculer un indice saprobique à partir de valences et de valeurs indicatrices attribuées à chaque espèce. Mais, dans le cas des invertébrés, l'identification jusqu'au niveau spécifique est très difficile, voire impossible pour certaines larves, à moins d'en posséder des stades adultes. L'indice saprobique n'est donc pratiquement jamais utilisé.

En raison de cette difficulté, certains auteurs ont élaboré des méthodes simplifiées, ne nécessitant pas de détermination spécifique. Nous avons définitivement écarté la méthode des indices biotiques (VERNEAUX et TUFFERY, 1967) qui donnait des résultats très aléatoires.

Nous appliquons ici la dernière évolution de cette méthode, à savoir l'indice biologique global normalisé ou IBGN (AFNOR, 1992).

La valeur indicielle est obtenue à partir d'un tableau à double entrée et dépend de la richesse faunistique (classe de variété) et du repérage de groupes indicateurs, classés de 9 à 1 suivant une échelle de résistance croissante à la pollution. Tous les organismes sont identifiés jusqu'au niveau de la famille sauf les oligochètes (sous-classe), les hydracariens (sous-ordre), les nématodes, les spongiaires, les bryozoaires et les némertiens (phylum).

Nous renvoyons à AGENCE DE L'EAU (1995), pour l'excellente analyse critique de cette méthode. Néanmoins, nous rappelons ici ses principaux inconvénients méthodologiques, qui imposent une grande prudence lors de l'interprétation des valeurs indicielles :

- on prend en compte la diversité comme critère de qualité, ce qui n'est pas vérifié pour des eaux oligo-dystrophes naturellement peu diversifiées ou des eaux eutrophiquées avec de grands herbiers riches en invertébrés;

- on ne considère pas quantitativement la structure du peuplement ;

- on ne peut identifier que les familles, ce qui conduit à grouper des espèces d'autoécologies pourtant différentes; 
- ces indicateurs sont surtout sensibles à l'oxygène et au type de substrat : l'indice ne mesure donc pas exclusivement la pollution;

- le protocole d'échantillonnage (prélèvements dans 8 biotopes différents par station) est difficile, voire impossible, à mettre en oeuvre dans des cours d'eau de petit gabarit.

A ce propos, comme il n'était pas possible de trouver ce nombre de biotopes dans toutes nos stations, nous avons préféré standardiser l'échantillonnage en adoptant une méthode simplifiée (DE PAUW et VANHOOREN, 1983) : pêche au troubleau pendant 1 minute en zone lotique, 1 minute en zone lentique et 1 minute en zone intermédiaire, les trois prélèvements étant réunis. Cette procédure rapide suffit généralement à prospecter les différents biotopes des petits cours d'eau.

L'IBGN varie de 20 à 1 par unité et est réparti en 5 classes (tableau I).

\subsection{Réalisation des figures 5 et 6}

Ces figures de synthèse rassemblent, pour 4 stations, les résultats des 4 types d'analyse : diagrammes ioniques, IPO, diatomées et invertébrés.

Des cercles de diamètre proportionnel aux teneurs sont donnés pour l'ammonium, les nitrites et les orthophosphates.

Pour les diatomées, on reprend la valeur de l'indice et le niveau de pollution, et on illustre la composition des peuplements par un dessin schématique des taxons les mieux représentés dans chaque catégorie (oligosaprobes en vert, $\beta$-mésosaprobes en jaune, $\alpha$-mésosaprobes en orange et polysaprobes en rouge). Les saproxènes, qui devraient être représentés en bleu, sont cependant si peu abondants ici qu'ils ont été groupés avec les oligosaprobes. Chaque individu dessiné correspond à plus ou moins $5 \%$ d'abondance relative (par exemple, 3 dessins pour $13 \%$ et 2 pour $12 \%$ ), le nombre total étant ajusté à $20(100 \%)$ pour chaque comptage. Dans le cas de peuplements très peu diversifiés, certains taxons, plus faiblement représentés mais ayant une signification écologique importante, sont aussi pris en compte (par exemple, Cymbella sinuata en A3 : 1 individu représenté pour 1,6\% d'abondance).

Pour les macro-invertébrés, on reprend la valeur de l'indice et le niveau de pollution, et on les représente également en fonction de leur abondance et de leur écologie : en se référant au tableau de I'IBGN (AGENCE DE L'EAU, 1995), nous avons représenté en bleu les groupes indicateurs 9 et 8 , en vert les groupes 7 et 6 , en jaune les groupes 5 et 4 , en orange les groupes 3 et 2 , et en rouge les groupes 2 et 1 .

\section{RÉSULTATS}

\subsection{Chimie des eaux (tableau II)}

\subsubsection{Minéralisation naturelle et eutrophisation (figure 2)}

Pour les affluents, nous représentons la Rulles supérieure (A1) sur substrat siliceux du Siegenien et le ruisseau des Alleines (A10) sur Siegenien et Gedinnien, avec respectivement 1,0 et 2,0 méq/l.

La Rulles supérieure appartient au type ardennais faiblement minéralisé (type oligotrophe). Nous classons le ruisseau des Alleines en ardennais à tendance mésotrophe.

Si la plupart des cours d'eau s'eutrophisent de l'amont vers l'aval par augmentation naturelle des teneurs en calcium, magnésium, potassium et bicarbonates-carbonates, on observe, dans la Semois, le phénomène inverse. Cette observation a déjà été réalisée par SYMOENS (1957). Cette situation originale apparaît bien sur les diagrammes ioniques que nous représentons pour quelques stations $(\mathrm{S} 1, \mathrm{~S} 3, \mathrm{S4}, \mathrm{S} 5, \mathrm{~S} 8, \mathrm{S12})$ auxquelles correspondent les minéralisations suivantes, exprimées en méq/l : 15,0 - 12,3 - 9,2 - 6,9-4,8 - 4,3 (figure 2).

Ainsi, la haute Semois est typiquement calcaire, puis on observe une diminution de la minéralisation, accentuée en aval de la Rulles et de la Vierre qui apportent des eaux plus pauvres. 
Mais il est remarquable de noter que les proportions ioniques restent les mêmes, l'allongement vertical du diagramme montrant la prédominance relative du calcium et des bicarbonates sur tout le cours. Cela se traduit, au niveau de la flore diatomique, par la présence de taxons alcaliphiles, même dans le cours inférieur où les teneurs ne sont plus très élevées et correspondent en fait au type famennien, intermédiaire entre les types ardennais et condrusien.

Tableau II : Analyses chimiques et indices de pollution organique (IPO).

Table II : Chemical analyses and organic pollution indexes (IPO).
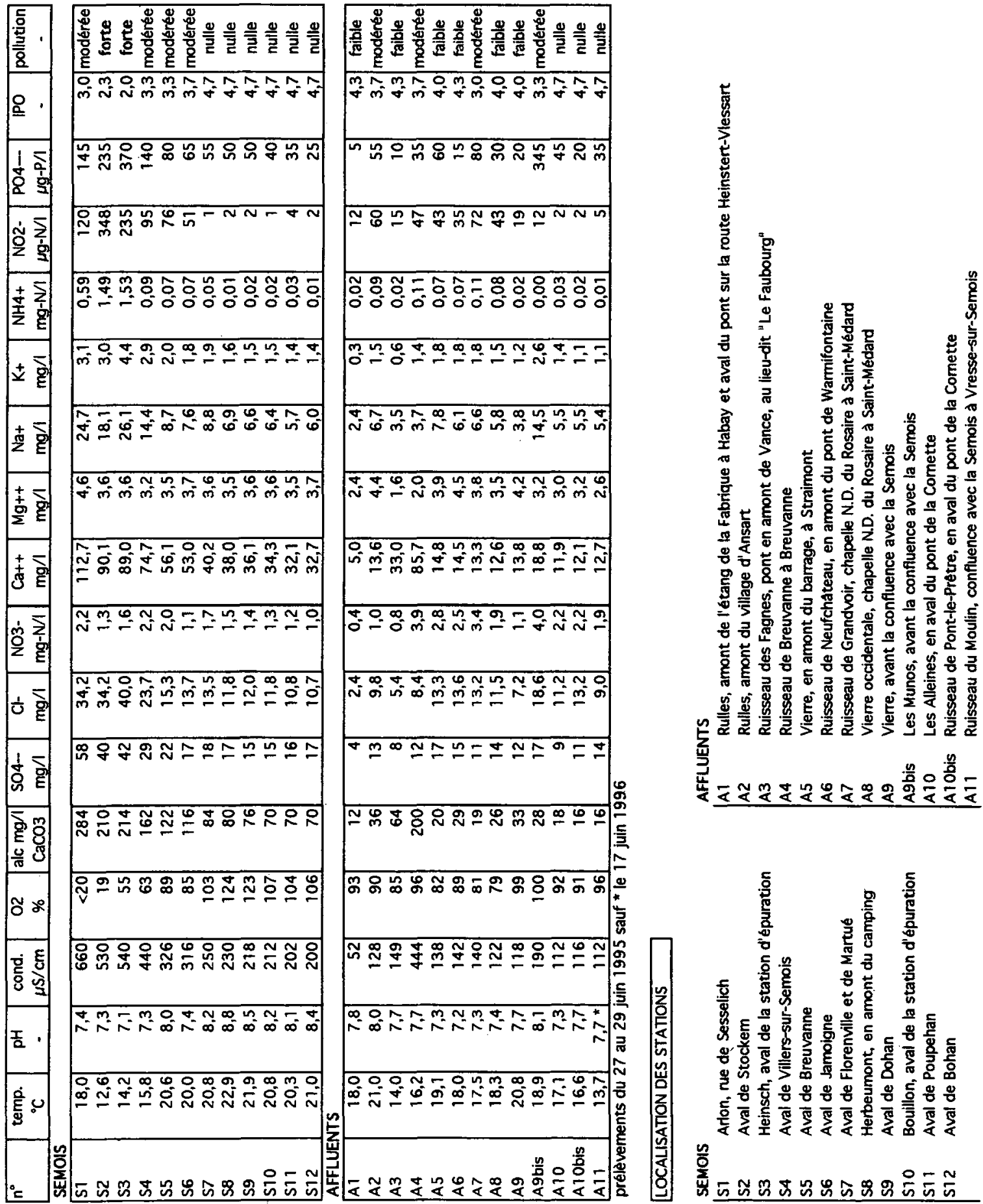


\section{SEMOIS}

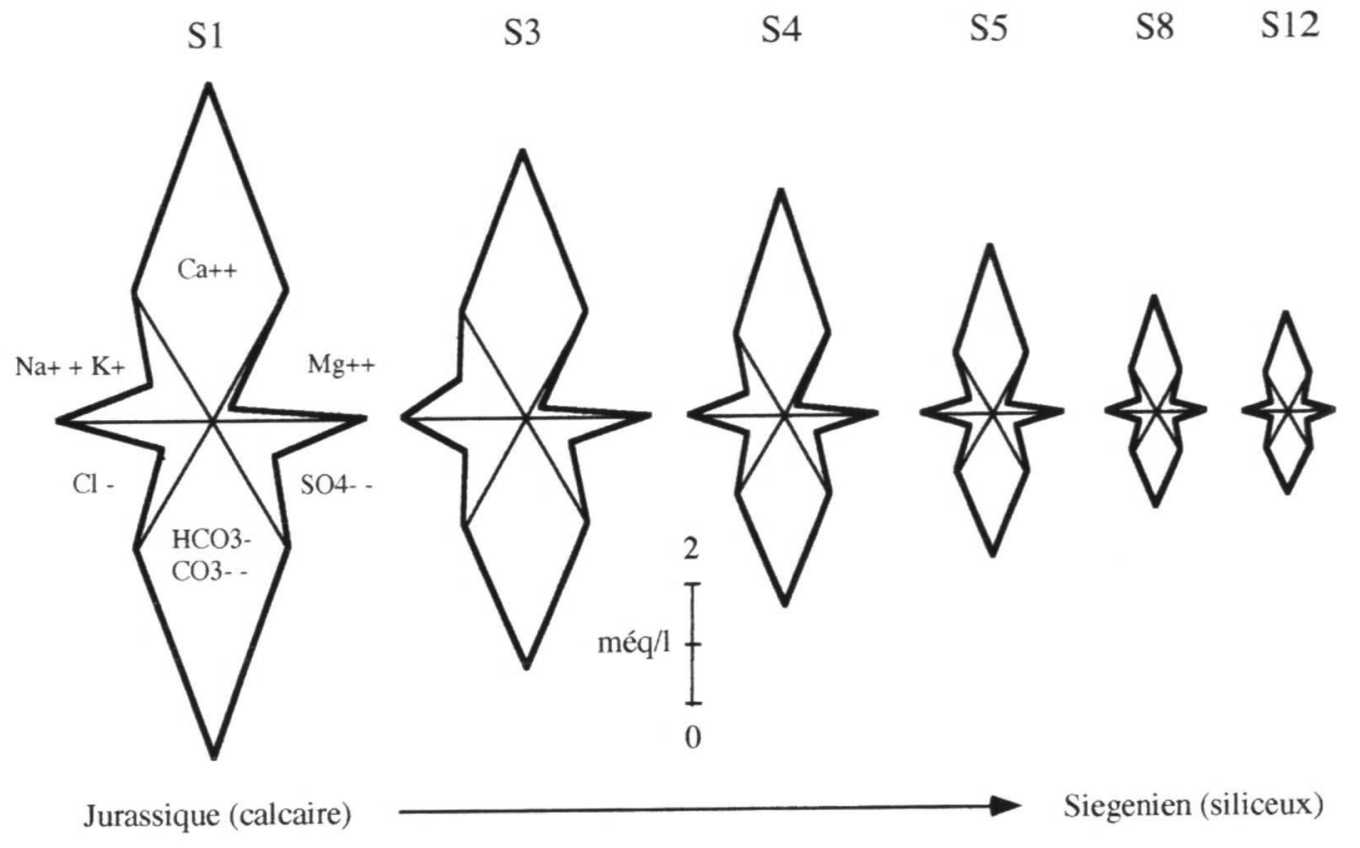

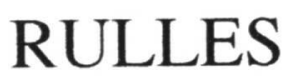

A1

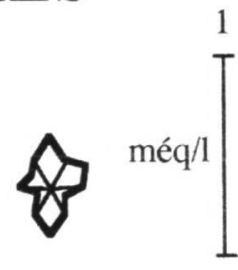

Siegenien (siliceux)
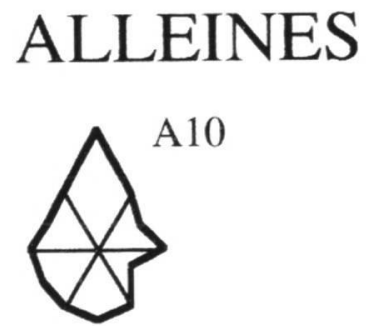

Siegenien + Gedinnien (siliceux)

Figure 2 : Diagrammes ioniques de 6 stations sur la Semois et de 2 affluents (Rulles et Alleines), et assises géologiques concernées.

Figure 2 : Ionic diagrams of 6 stations in the Semois and 2 tributaries (Rulles and Alleines), with their geological status.

II ne s'agit donc pas d'une situation simplement inverse à celle de l'eutrophisation qui suppose le passage progressif du type fagnard ou ardennais vers le type condrusien, avec modification très nette des proportions ioniques et des types de peuplements algaux.

\subsubsection{Niveaux d'eutrophication}

Pour la Semois, la teneur en oxygène dissous fixe nettement, entre S6 et S7, la transition entre les stations poliuées et les stations eutrophiquées (tableau II). On passe d'un déficit dû à la présence de matières organiques en $\mathrm{S} 6$ à la saturation en $\mathrm{S} 7$, puis à la sursaturation en $\mathrm{S} 8$ et S9. Les teneurs en nitrates et orthophosphates sont assez faibles, car assimilées par.la biomasse végétale. Cette assimilation n'est pas suffisante pour faire tomber les teneurs en orthophosphates sous le seuil des $15 \mu \mathrm{g}-\mathrm{P} / \mathrm{l}$.

La biomasse est surtout constituée de vastes peuplements de Ranunculus fluitans Lam. et d'algues filamenteuses qui participent à l'autoépuration. 
Parmi les affluents, les eaux des stations A4 à A8 sont plus riches en nitrates que celles de la moyenne et de la basse Semois, mais ces cours d'eau, sous couvert forestier, ne réunissent pas les conditions favorables à un fort développement végétal. D'autre part, les teneurs en nitrites sont plus élevées que dans la Semois inférieure : la nitrification y serait plus lente.

Une biomasse algale importante caractérise les stations A9 et A9bis, qui sont nettement eutrophiquées. Les stations A1, A3, A10, A10bis et A11 sont normales.

\subsubsection{Niveaux de pollution}

Les valeurs de I'IPO et les niveaux de qualité correspondants sont donnés dans le tableau II, dans les deux dernières colonnes, et sont portés sur la figure 3 avec le code de couleur.

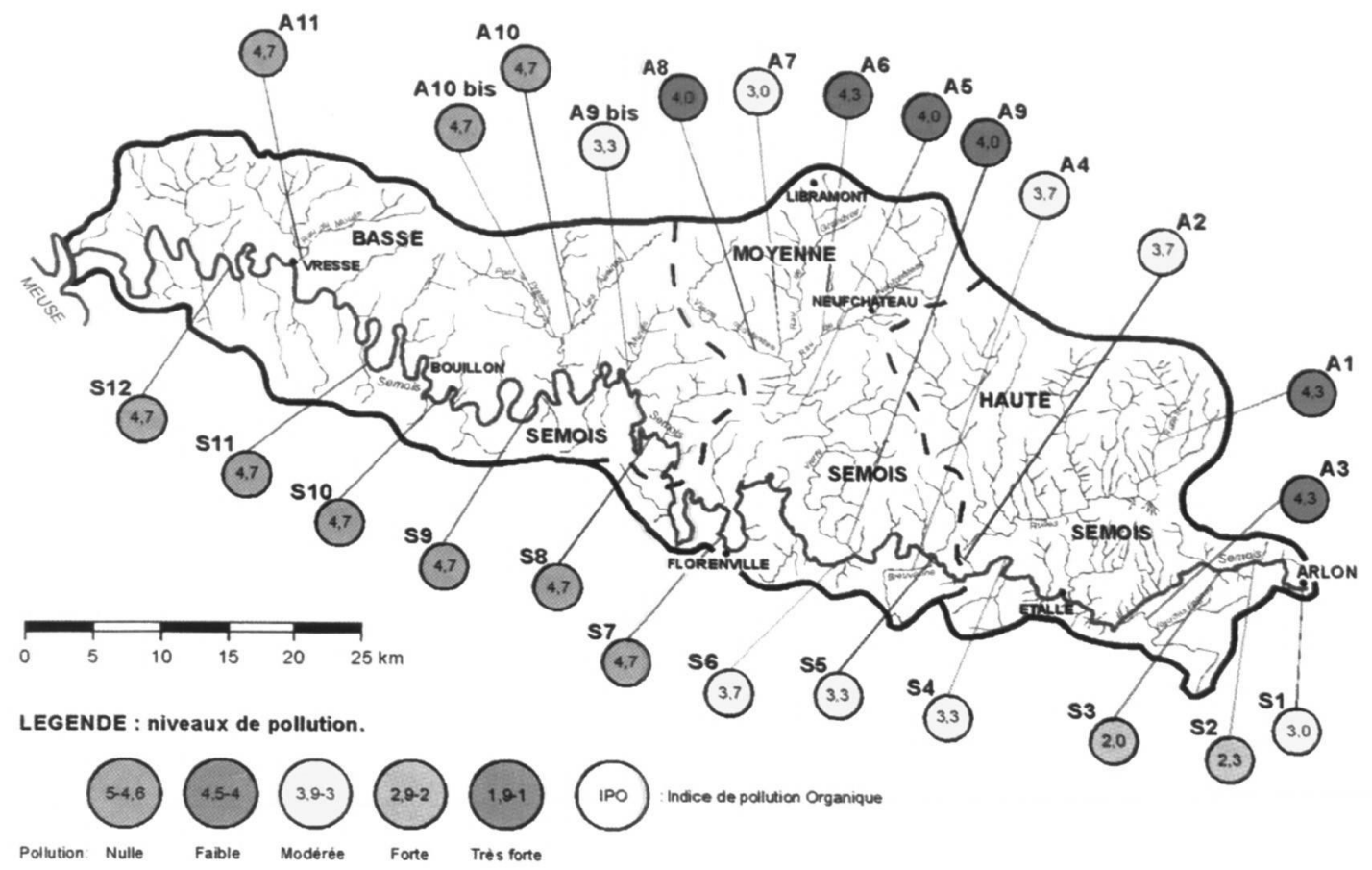

Figure 3 : Carte de la Semois avec les IPO (toutes les stations de la Semois en-dessous de la carte, les affluents au-dessus).

Figure 3 : Map of the Semois catchment area with the IPO values (the Semois stations below, the tributaries at the top).

L'IPO est de 3,0 (pollution modérée) à la première station de la Semois. Ce résultat ne concorde pas avec la situation catastrophique observée lors du prélèvement : une couche d'hydrocarbures recouvrait l'ensemble du lit. Pour cette raison, l'oxygène n'a pu être mesuré avec précision : on était probablement en anaérobiose, les sédiments étant noirs et en état de fermentation. Dans ces conditions, il faut craindre des interférences dans l'analyse de certains paramètres ; de plus, la minéralisation des matières organiques est probablement ralentie et son effet n'apparaît qu'aux deuxième et troisième stations (pollution forte), laquelle reçoit en plus le rejet très minéralisé de la station d'épuration à Heinsch. L'indice passe ainsi à 2,3 puis à 2,0.

Ensuite, I'IPO remonte rapidement pour atteindre 4,7 (pollution nulle) à partir de Martué (S7). Ces bonnes valeurs s'expliquent par les très faibles teneurs en ammonium et en nitrites, mais masquent la présence d'orthophosphates en quantité supérieure à la limite de l'eutrophication. 
Parmi les affluents importants, la Rulles (A2) apporte une eau modérément polluée, ce qui est un élément nouveau car, auparavant (ROSILLON, comm. pers.), les eaux de ce cours d'eau venaient plutôt diluer la pollution de la Semois. La Vierre (A9), par contre, apporte des eaux faiblement polluées, qui font passer l'IPO des eaux de la Semois brusquement de 3,7 (pollution modérée) à 4,7 (pollution faible). On soulignera le rôle épurateur positif de la station d'épuration de Neufchâteau et du barrage sur la Vierre. Les trois derniers affluents, coulant dans un contexte forestier (A10, A10bis et A11), sont de bonne qualité et participent à l'amélioration de la basse Semois.

\subsection{Biologie des eaux}

\subsubsection{Les diatomées benthiques}

\subsubsection{Préliminaires}

149 taxons de diatomées ont été identifiés dans les 25 stations. Le tableau III reprend les relevés complets, exprimés en pourcentage d'abondance relative, ainsi que les valences saprobiques (vs) et valeurs indicatrices (vi) de notre indice (LECLERCQ et MAQUET, 1987, modifié et complété).

Pour les patronymes de ces taxons, on se reportera à la flore de KRAMMER et LANGEBERTALOT (1986-1991) et, pour les Pinnularia, à la publication de KRAMMER (1992). Quelques changements taxonomiques plus récents ne sont donc pas pris en compte. Nous maintenons cependant deux dénominations plus anciennes. D'une part, Fragilaria capucina Desm. var. lanceolata Grun. est un taxon assez petit, lancéolé, avec des extrémités aiguës, et des stries serrées et peu visibles. Nous le trouvons systématiquement dans les eaux pures oligotrophes de l'Ardenne et nous lui attribuons une grande importance comme indicateur typologique et de qualité (FABRI et LECLERCQ, 1984, planche 16, fig. 460-462) ; aucune illustration de la littérature ne lui correspond fidèlement, si ce n'est le dessin de HUSTEDT (1927-1966, fig. 659 f-g). D'autre part, nous maintenons une distinction entre Pinnularia subcapitata et $P$. hilseana mis en synonymie par KRAMMER (1992) : en effet, nos résultats anciens (FABRI et LECLERCQ, 1984) et postérieurs, indiquant que $P$. hilseana est nettement plus acidophile que $P$. subcapitata, prônent pour le maintien de cette distinction.

Le tableau III est exploité de trois manières : un tableau écologique, l'indice diatomique cartographié et une figure de synthèse représentant quantitativement les taxons importants.

Le tableau écologique (tableau IV) totalise, pour chaque station, les pourcentages atteints par les espèces de même auto-écologie soit, dans le cas présent, 5 catégories, conformément à la méthode de l'indice diatomique:

- taxons saproxènes d'eau non polluée (valence saprobique entre 5,0 et 4,3 ) ;

- taxons oligosaprobes d'eau faiblement polluée (vs entre 4,2 et 3,6) ;

- taxons $\beta$-mésosaprobes d'eau modérément polluée (vs entre 3,5 et 3,0 ) ;

- taxons $\alpha$-mésosaprobes d'eau fortement polluée (vs entre 2,9 et 2,3 ) ;

- taxons polysaprobes d'eau très fortement polluée (vs entre 2,2 et 1,0 ).

Ce tableau donne aussi la valeur de l'indice diatomique calculé à partir de tous les taxons présents et les niveaux de pollution correspondants.

L'indice est cartographié, avec les couleurs correspondantes (figure 4).

Tableau III : Relevés de diatomées dans les 25 stations. Résultats en pourcentages d'abondance relative. Colonne $2:$ vs $=$ valence saprobique $;$ colonne $3:$ vi = valeur indicatrice. Nomenclature : voir chapitre 3.2.1.1.

Table III : Diatoms assemblages in the 25 stations. Results in percentages of relative abundance. In column 2 : vs = saprobial value ; in column $3:$ vi = indicative weight. Nomenclature : see chapter 3.2.1.1. 


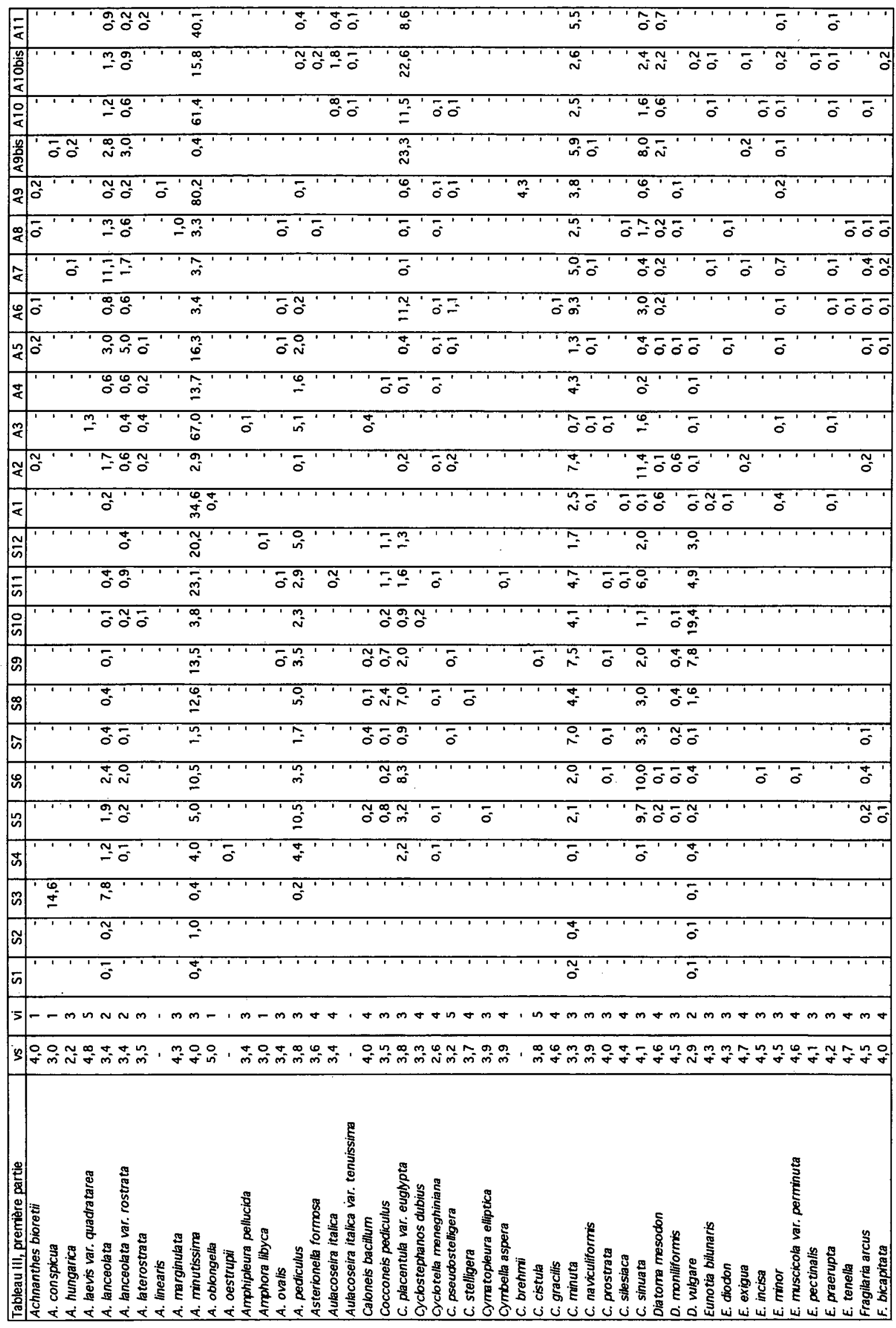




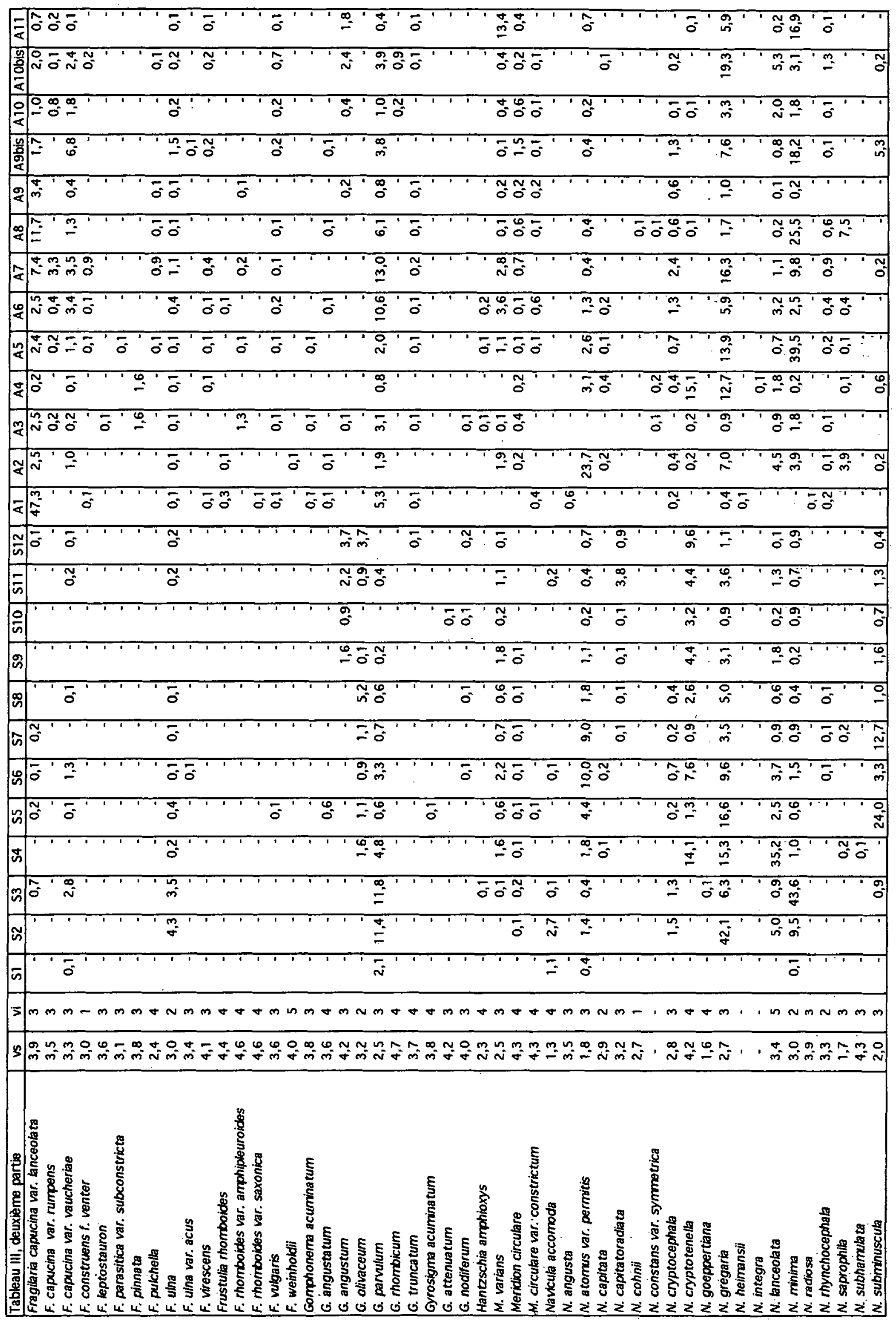




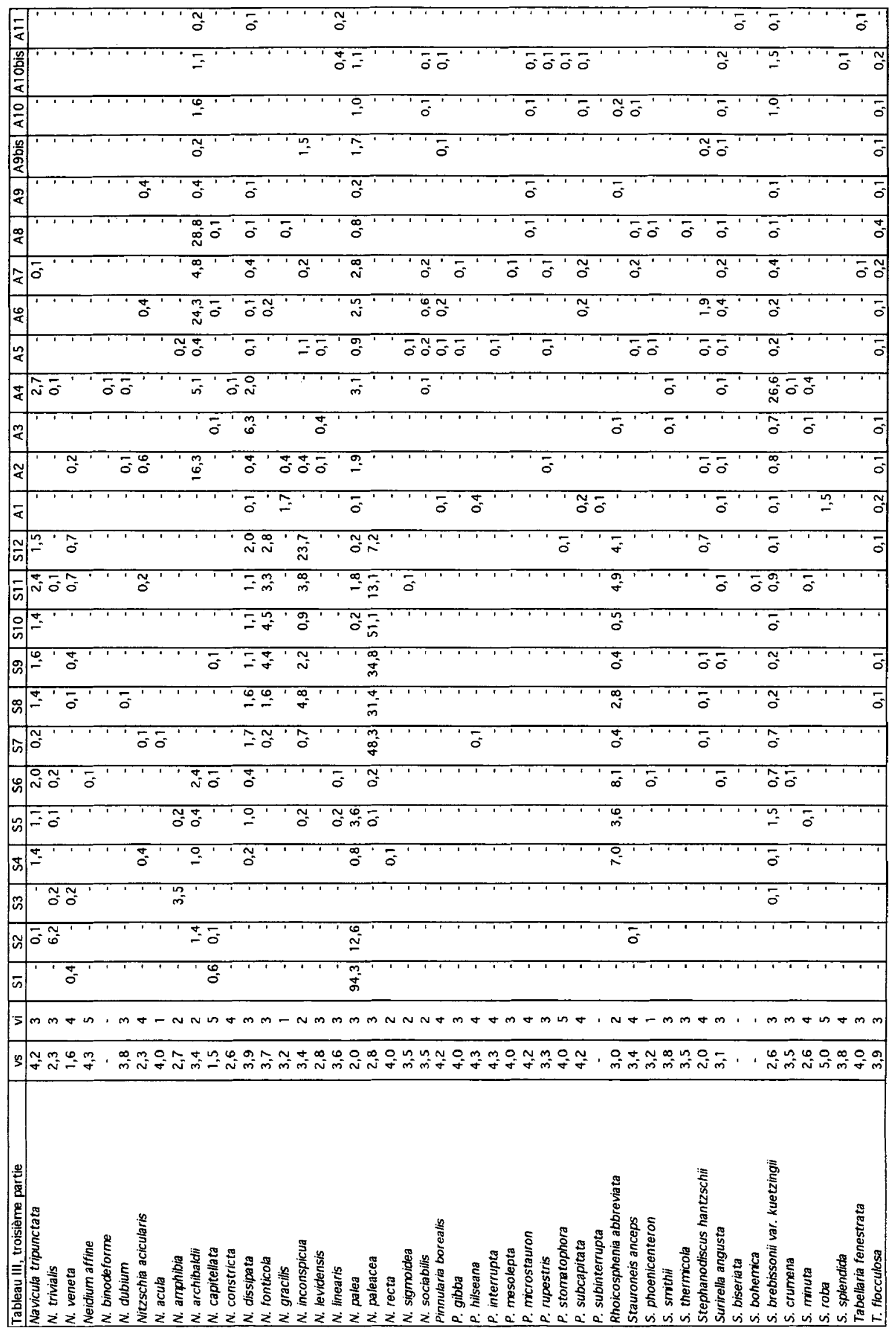


Les figures de synthèse (figures 5 et 6 ) illustrent les taxons les mieux représentés dans chaque catégorie caractérisée par les couleurs de référence (règles pour le tracé de ces figures : chapitre 2.3.).

\subsubsection{Typologie des peuplements naturels de diatomées}

Sur les 25 stations, 5 sont dominées par les taxons sensibles, saproxènes et oligosaprobes (tableau IV), et correspondent à différents niveaux typologiques de référence.

Tableau IV : Synthèse des comptages de diatomées (abondances relatives cumulées en \%) et indices diatomiques.

Table IV : Synthesis of the diatoms counts (cumulate relative abundances in \%) and diatom indexes.

\begin{tabular}{|c|c|c|c|c|c|c|c|c|c|c|c|c|}
\hline SEMOIS & & & & & & & & & & & & \\
\hline Taxons & S1 & S2 & 53 & 54 & S5 & TS6 & TS7 & 58 & 59 & S10 & S11 & S12 \\
\hline $\begin{array}{l}\text { saproxènes } \\
\text { oligosaprobes } \\
\text { B-mésosaprobes } \\
\text { alpha-mésosaprobes } \\
\text { polysaprobes }\end{array}$ & $\begin{array}{r}0,0 \\
0,4 \\
0,5 \\
2,2 \\
96,9 \\
\end{array}$ & $\begin{array}{r}0,1 \\
1,1 \\
20,8 \\
61,3 \\
16,7 \\
\end{array}$ & $\begin{array}{r}0,2 \\
1,3 \\
73,3 \\
23,5 \\
1,7 \\
\end{array}$ & $\begin{array}{r}0,2 \\
26,6 \\
47,5 \\
22,8 \\
2,9 \\
\end{array}$ & $\begin{array}{r}0,7 \\
33,3 \\
13,8 \\
20,2 \\
32,0 \\
\end{array}$ & $\begin{array}{r}1,1 \\
42,8 \\
25,1 \\
17,5 \\
13,5 \\
\end{array}$ & $\begin{array}{r}0,5 \\
11,4 \\
12,0 \\
54,2 \\
21,9 \\
\end{array}$ & $\begin{array}{r}0,5 \\
35,3 \\
21,4 \\
39,8 \\
3,0 \\
\end{array}$ & $\begin{array}{r}0,6 \\
34,7 \\
13,5 \\
47,9 \\
3,3 \\
\end{array}$ & $\begin{array}{r}0,1 \\
19,4 \\
7 ; 6 \\
71,8 \\
1,1 \\
\end{array}$ & $\begin{array}{r}0,1 \\
47,4 \\
23,5 \\
24,5 \\
4,5 \\
\end{array}$ & $\begin{array}{r}0,0 \\
48,8 \\
37,1 \\
11,5 \\
2,6 \\
\end{array}$ \\
\hline $\begin{array}{l}\text { Indice diatomique } \\
\text { Pollution ** }\end{array}$ & $\frac{2,0}{T F}$ & $\frac{2,6}{F}$ & $\frac{2,9}{F}$ & $\begin{array}{c}3,4 \\
\mathrm{~m}\end{array}$ & $\begin{array}{l}3,0 \\
\mathrm{~m}\end{array}$ & $\begin{array}{l}3,3 \\
m\end{array}$ & $\frac{2,8}{F}$ & $\begin{array}{l}3,3 \\
\mathrm{~m}\end{array}$ & $\begin{array}{l}3,3 \\
\mathrm{~m}\end{array}$ & $\begin{array}{l}3,1 \\
\mathrm{~m}\end{array}$ & $\begin{array}{l}3,5 \\
\mathrm{~m}\end{array}$ & $\begin{array}{c}3,6 \\
f\end{array}$ \\
\hline
\end{tabular}

\section{AFFLUENTS}

\begin{tabular}{|c|c|c|c|c|c|c|c|c|c|c|c|c|c|}
\hline Taxons des eaux & A1 & $\mathrm{A2}$ & $\overline{\mathrm{A3}}$ & A4 & $\overline{\mathrm{A} 5}$ & $\widehat{A 6}$ & A7 & $\widehat{A 8}$ & $\mathrm{Ag}^{*}$ & A9bis & A10 & \begin{tabular}{|l} 
A10 \\
bis
\end{tabular} & $\overline{\mathrm{A} 11}$ \\
\hline sapr & 4,6 & 1,4 & 3,2 & 0,2 & 1,0 & 1,5 & 2,4 & 2,5 & 0,8 & 4,0 & 1,9 & 3,8 & $\overline{1,2}$ \\
\hline oligosaprobes & 83,6 & 18,3 & 86,0 & $\underline{37,6}$ & 22,8 & 22,0 & 14,1 & 18,2 & 85,9 & 34,3 & 76,6 & 48,2 & 53,2 \\
\hline B-mésosaprobes & 5,6 & 37,1 & 5,2 & $\overline{13,5}$ & $\underline{53,9}$ & 47.7 & 44,1 & 61,6 & 5,7 & 41,1 & $\overline{14,2}$ & $\overline{20 ; 8}$ & $\overline{25,1}$ \\
\hline alpha-mésosaprobes & 6,1 & $\overline{13,2}$ & 5,5 & 41,7 & 18,7 & 22,6 & 35,8 & 8,9 & 3,3 & $\overline{12,8}$ & 6,0 & 25,8 & 19,8 \\
\hline polysaprobes & 0,1 & 30,0 & 0,1 & 7,0 & 3,6 & 6,2 & 3,6 & 8,8 & 0,2 & 7,8 & 1,3 & 1,4 & 0,7 \\
\hline Indic & 3,8 & 2,9 & 3,9 & $\overline{3,3}$ & 3,2 & 3,2 & 3,1 & 3,2 & $\overline{3,9}$ & 3,3 & 3,8 & 3,5 & 3,5 \\
\hline Pollution ** & 18 & $F$ & $f$ & $\mathrm{~m}$ & $\mathrm{~m}$ & $\mathrm{~m}$ & $\mathrm{~m}$ & $m$ &, 0 & $\mathrm{~m}$ & 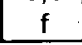 & $\mathrm{m}$ & $\mathrm{m}$ \\
\hline
\end{tabular}

\section{Remarques}

pour la Semois, groupe dominant en caractères gras soutignés

* en A9, le total est de $95,9 \%$ car Cymbella brehmii, taxon rare, n'a pas encore de valence saprobique.

** pollution : $n$ : nulle; $f$ : faible ; $m$ : modérée ; $F$ : forte; TF : très forte.

La Rulles en amont (A1) est dominée par Achnanthes minutissima et Fragilaria capucina var. lanceolata, accompagnés par Achnanthes oblongella, Diatoma mesodon, Eunotia minor, Meridion circulare var. constrictum, Navicula angusta, Nitzschia gracilis, Surirella roba : ce peuplement caractérise les eaux de «type ardennais oligotrophe ». Ce cours d'eau est en contact uniquement avec le Siegenien.

La Vierre en aval (A9) est caractérisée par un peuplement proche de celui de la Rulles (A1), dominé par Achnanthes minutissima et quelques Fragilaria capucina var. lanceolata, mais accompagnés par Cocconeis placentula var. euglypta et Cymbella sinuata qui indiquent une eau un peu plus minéralisée. Ce cours d'eau est en contact principalement avec le Siegenien, mais certaines sources sont sur le Gedinnien.

Le ruisseau des Alleines (A10) est dominé par Achnanthes minutissima, Cocconeis placentula var. euglypta et quelques Cymbella sinuata; accompagnés par Diatoma mesodon, Fragilaria capucina var: lanceolata, Gomphonema angustum, Meridion circulare : ce peuplement indique une mésotrophie plus accusée: ("type ardennais à tendance mésotrophe.»). Ce cours 
d'eau est pour moitié sur le Siegenien et sur le Gedinnien ; le ruisseau de Pont-le-Prêtre (A10bis), dans une situation géologique semblable, et le ruisseau du Moulin (A11) uniquement sur Gedinnien, présentent le même type de peuplement, mais sont légèrement altérés.

Le ruisseau des Fagnes (A3) est dominé par Achnanthes minutissima, accompagné de Fragilaria capucina var. lanceolata, Cymbella sinuata, Amphora pediculus et Nitzschia dissipata, accompagnés par Caloneis bacillum, Fragilaria pinnata, Frustulia rhomboides var. amphipleuroides et Meridion circulare : ce peuplement correspond à des eaux plus riches (" type ardennais mésotrophe "). Ce cours d'eau, sur les roches du Jurassique, devrait pourtant être de type calcaire. Cependant, dans cette région, il y a eu une décalcification totale des grès et de grands dépôts de sables siliceux : ce ruisseau est donc alimenté par des nappes peu minéralisées et peut être aussi en contact localement avec des roches calcaires, ce qui explique le niveau de minéralisation intermédiaire.

La Semois (S12) est dominée par Achnanthes minutissima, accompagné de Navicula cryptotenella, Amphora pediculus, Cymbella sinuata, Gomphonema angustum, Nitzschia fonticola, Cocconeis pediculus, C. placentula var. euglypta, Diatoma vulgare, Gomphonema olivaceum, Navicula capitatoradiata, N. tripunctata, Nitzschia dissipata, Rhoicosphenia abbreviata : tous ces taxons indiquent une plus grande richesse en calcium et leurs proportions correspondent au " type famennien ", intermédiaire entre les types ardennais siliceux et condrusien calcaire. La géologie particulière du cours de la Semois (voir introduction) explique aisément ce peuplement de diatomées.

Pour compléter cette description typologique, nous ajoutons le ruisseau de Breuvanne (A4). Bien qu'il soit touché par une pollution modérée (Surirella brebissonii var. kuetzingii, Navicula gregaria, Navicula atomus var. permitis, ...), son peuplement est encore dominé par des espèces caractéristiques: Achnanthes minutissima et Navicula cryptotenella, accompagnés par N. tripunctata, Amphora pediculus, Fragilaria pinnata et Nitzschia dissipata. C'est notamment l'abondance de Navicula cryptotenella $(15,1 \%)$ qui permet de rattacher ce ruisseau au " type condrusien " aux eaux calcaires. Son cours est entièrement sur le Jurassique.

Ce classement typologique des peuplements de diatomées peut être comparé avec la conductivité, mesure globale de la minéralisation. On mesure une conductivité de $52 \mu \mathrm{S} / \mathrm{cm}$ pour le type ardennais oligotrophe (A1), de 112 à $118 \mu \mathrm{S} / \mathrm{cm}$ pour le type ardennais à tendance mésotrophe (A9, A10, A10bis et $A 11)$, de $149 \mu \mathrm{S} / \mathrm{cm}$ pour le type ardennais mésotrophe (A3), de $200 \mu \mathrm{S} / \mathrm{cm}$ pour le type famennien (S12) et de $444 \mu \mathrm{S} / \mathrm{cm}$ pour le type condrusien (A4).

En conclusion, les stations naturelles du bassin de la Semois sont toutes dominées par Achnanthes minutissima, taxon caractérisant des eaux non ou peu polluées, mais à très large amplitude écologique du point de vue de la minéralisation. On trouve, à côté, différents taxons moins abondants, mais importants, car ce sont eux qui indiquent les niveaux trophiques. Cinq assemblages de diatomées sont décrits; ils correspondent à une conductivité croissante, surtout influencée par le calcium dans des conditions naturelles. Une relation étroite existe entre ces assemblages et la géologie du bassin versant : plus particulièrement pour le Dévonien inférieur, le type ardennais à tendance mésotrophe apparaît chaque fois qu'il y a un contact avec le Gedinnien, assise plus riche que le Siegenien comme nous l'avons déjà montré précédemment (LECLERCQ, 1984).

La grande sensibilité des diatomées vis-à-vis des facteurs naturels d'eutrophisation (calcium, magnésium, bicarbonates, ...) en fait un matériel de choix pour les études typologiques de référence.

\subsubsection{3. Étude des pollutions (tableaux III et IV, figure 4)}

La Semois est touchée par la pollution organique, dès sa source.

A la station S1, l'indice diatomique (ID) est de 2,0 (pollution très forte) et le peuplement est largement dominé par les taxons polysaprobes (Nitzschia palea : 94,3 \%). L'ID augmente ensuite à 2,6 en $\mathrm{S} 2$ : le peuplement est alors dominé par les taxons $\alpha$-mésosaprobes 
(Gomphonema parvulum, Navicula gregaria). Ce premier tronçon fortement pollué est dû aux rejets non épurés d'une partie de la ville d'Arlon, principalement la zone industrielle.

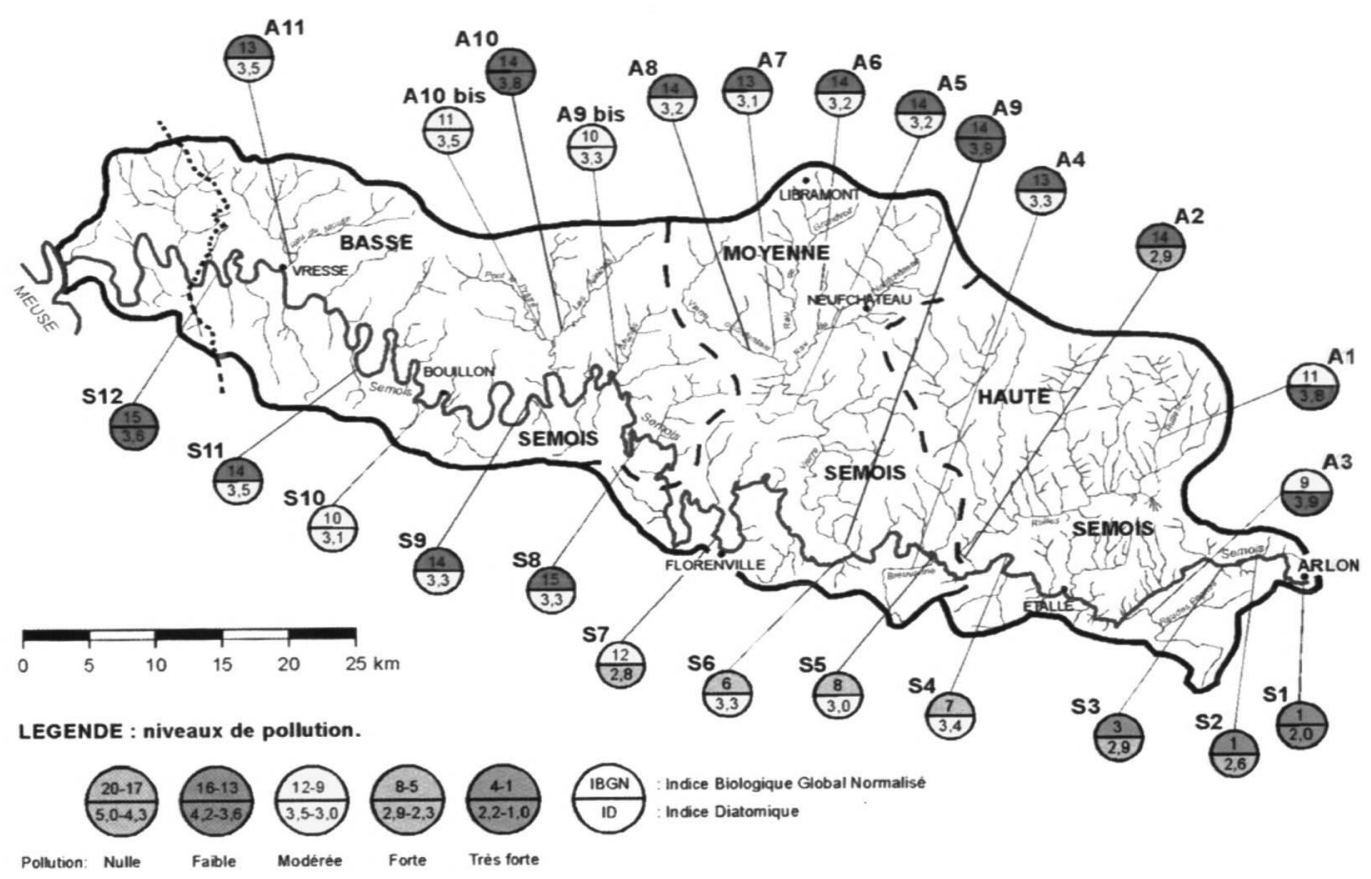

Figure 4 : Carte de la Semois avec les ID et IBGN (toutes les stations de la Semois en-dessous de la carte, les affluents au-dessus).

Figure 4 : Map of the Semois basin with the ID and IBGN (the Semois stations below, the tributaries at the top).

La station S3 est toujours fortement polluée (ID $=2,9$ ). Elle reçoit les eaux usées partiellement épurées par la station d'épuration d'Arlon, qui ne semble pas assez performante. Le peuplement diatomique est ici dominé par Navicula minima, que nous rencontrons systématiquement en aval des rejets de stations d'épuration à boues activées : nous pensons que la prolifération de cette espèce est liée à des teneurs élevées en nitrates et orthophosphates, résultant de la minéralisation des matières organiques. Dans ce sens, elle est une très bonne indicatrice de l'eutrophication causée par les stations d'épuration à boues activées qui réalisent bien la digestion aérobie (épuration secondaire), mais très peu l'assimilation des nutriments (épuration tertiaire), et qui rejettent donc des quantités importantes de nitrates et d'orthophosphates. Navicula minima peut aussi réagir à l'eutrophication venant du lessivage des amendements agricoles.

Les stations $\$ 4$, S5 et S6 sont modérément polluées, mais leurs peuplements diatomiques sont assez différents. S4 (ID $=3,4)$ est dominée par les taxons $\beta$-mésosaprobes, accompagnés par environ $20 \%$ de taxons oligosaprobes. II y a donc une nette amélioration. S5 présente un peuplement plus altéré $(I D=3,0)$, dominé simultanément par des taxons oligosaprobes et polysaprobes : ce mélange composite résulte de variations importantes dans les rejets pollués déversés dans la Rulles, laquelle présente aussi un peuplement composite de taxons $\beta$-mésosaprobes et de polysaprobes (A2). S6 s'améliore (ID $=3,3$ ) et est dominée par des taxons öligosaprobes et $\beta$-mésosaprobes.

En $S 7$, on repasse en pollution forte $(\mathrm{ID}=2,8)$, suite aux rejets non épurés de Florenville notamment : les diatomées dominantes sont $\alpha$-mésosaprobes. 
En S8 et S9, la Semois passe en pollution modérée : le peuplement est co-dominé par les taxons $\alpha$-mésosaprobes et oligosaprobes, indiquant une instabilité des conditions chimiques. Les rejets de différents villages entretiennent cette situation.

En S10, une nouvelle dégradation (ID = 3,1) survient, en aval de Bouillon. On peut mesurer ici l'effet d'une agglomération épurée ou non. En effet, Florenville n'est pas épurée et provoque le passage de la Semois en pollution forte (diminution de l'ID de 0,5 point, développement de polysaprobes). La ville de Bouillon est épurée et provoque une diminution de I'ID limitée à 0,2 point, sans développement de polysaprobes : on reste en pollution modérée, mais les taxons $\alpha$-mésosaprobes dominent car l'épuration n'est pas complète (station par boues activées, comme à Arlon).

Enfin, en $\mathrm{S} 11$ et $\mathrm{S} 12$, les taxons oligosaprobes dominent et les $\alpha$-mésosaprobes régressent : I'ID passe à 3,5 puis à 3,6 . C'est une Semois faiblement polluée, mais encore eutrophiquée ( $\beta$-mésosaprobes codominants), qui quitte le territoire belge.

Au niveau des affluents, la Rulles inférieure (A2) est fortement polluée (ID $=2,9$ ) ; son impact sur la Semois est bien marqué en $S 5$, cette dernière frôlant la pollution forte (ID $=3,0$ ). La Vierre et ses affluents ( $A 5$ à $A 8$ ) sont modérément altérés (ID $=3,1$ à 3,2 ) : il s'agit ici d'une eutrophication due à la station d'épuration de Neufchâteau (boues activées) et à l'agriculture. Cette eutrophication est cependant partiellement résorbée par un barrage, ce qui permet à la Vierre (A9) d'atteindre un niveau de pollution faible à sa confluence avec la Semois (ID = 3,9).

Le ruisseau des Munos (A9bis) est également eutrophiqué en aval de la station d'épuration de Bertrix, dont les effluents sont enrichis en orthophosphates et en azote minéral.

Les stations $A 1, A 3$ et $A 10$ sont proches de l'état naturel et amènent des eaux propres dans la Semois. Les stations A4, A10bis et A11 sont légèrement altérées. Pour rappel, les peuplements de ces stations ont été discutés au chapitre 3.2.1.2.

En conclusion, la Semois passe d'une pollution forte à une pollution faible, de l'amont vers l'aval, dans son parcours belge. Cette évolution est irrégulière, car elle est perturbée par des apports pollués qui viennent de certains affluents.

\subsubsection{Les macro-invertébrés benthiques}

\subsubsection{Préliminaires}

Dans les tableaux V et VI, on trouvera les résultats des prélèvements d'invertébrés dans la Semois et dans les affluents.

Plusieurs personnes ayant travaillé de façon quelque peu différente, il n'a pas été possible de standardiser les deux tableaux. Ainsi, dans le tableau VI, les nombres d'individus sont donnés exactement quand ils sont inférieurs à 10 et sont donnés simplement supérieurs à 10, au-delà ; les déterminations ont été faites jusqu'au genre, dans certains cas. Dans le tableau $V$, on cite les nombres $1,2,3$, supérieurs à 3 (et inférieurs à 10) et supérieurs à 10 ; les taxons sont tous identifiés jusqu'à la famille ou l'ordre. Cette différence ne modifie cependant pas la détermination des valeurs indicielles.

\subsubsection{2. Étude des pollutions (tableaux $V$ et VI, figure 4)}

Une pollution très forte est notée en $\mathrm{S} 1, \mathrm{~S} 2$ et $\mathrm{S} 3$ : le milieu, pratiquement anoxique et couvert de vase organique, ne convient qu'à quelques oligochètes et chironomes. Une légère amélioration est marquée en S3 par l'apparition de quelques Ancylidae et Gammaridae.

Une pollution forte touche les stations S4, S5 et S6: la diminution de son intensité se marque par l'apparition de quelques trichoptères à fourreau (Leptoceridae). En S6, la diminution de l'indice n'est probablement pas due à une diminution de la qualité de l'eau, mais plutôt au hasard du prélèvement : en effet, on n'y a trouvé que 2 Leptoceridae qui ne peuvent donc plus intervenir comme groupe indicateur, alors que 3 individus pourraient compter. 
Tableau V : Peuplements d'invertébrés de la Semois (résultats en nombres d'individus).

Table V : Invertebrates of the river Semois (results in numbers of organisms).

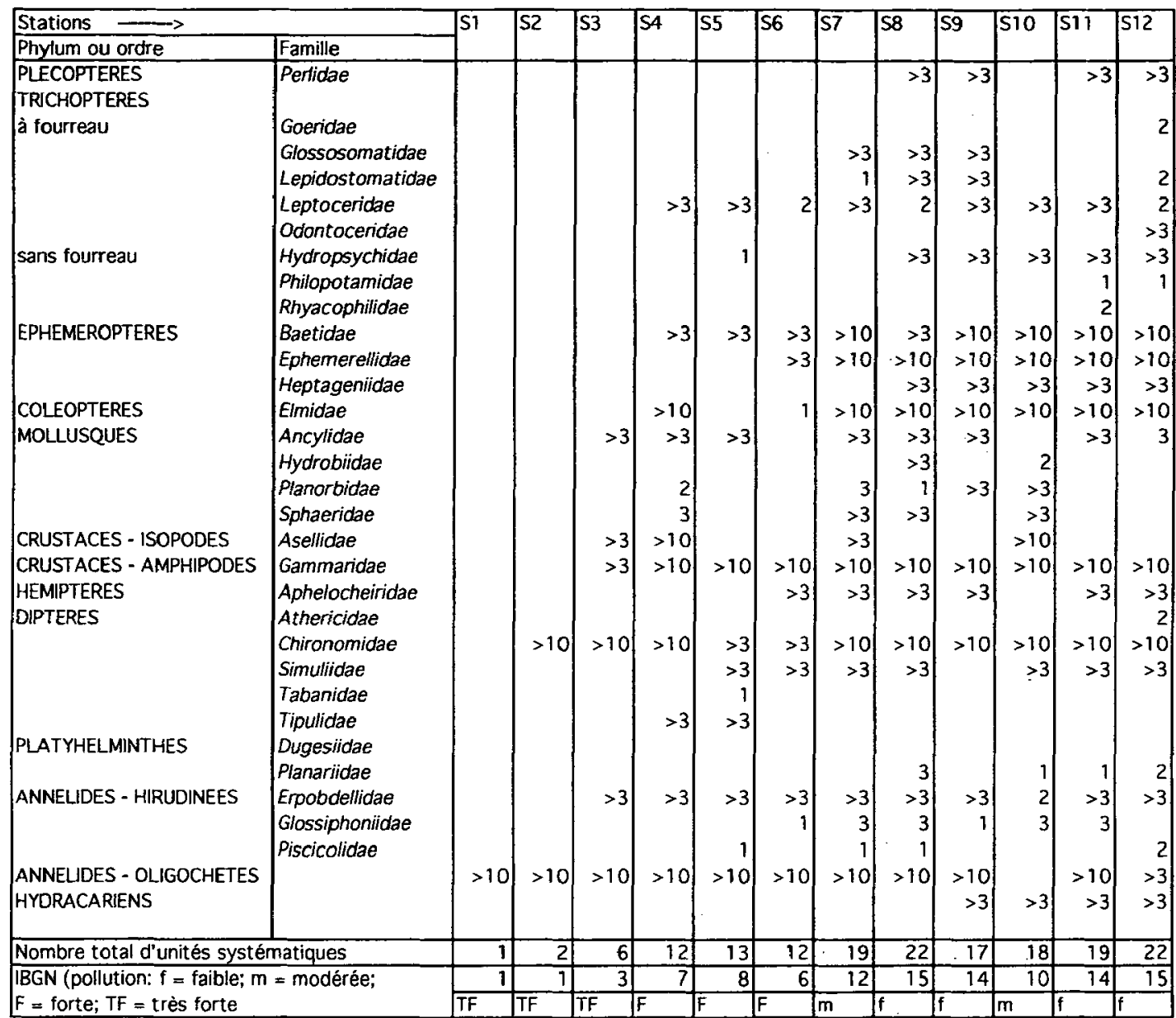

La chimie et les diatomées confirment ce diagnostic. Que l'on applique ou non le protocole de prélèvement de l'IBGN (visite de 8 biotopes), ce problème de limite à 3 individus reste un des défauts de la méthode qui explique son manque de sensibilité.

La station S7 reçoit un indice de 12 (pollution modérée). Le groupe indicateur est celui des Glossosomatidae (trichoptères à fourreau). Cette nouvelle amélioration suit celle de l'indice de pollution organique (IPO). Pourtant, les diatomées indiquent, au contraire et sans équivoque, une dégradation due aux rejets de Florenville. Or, nous avons dit plus haut qu'à partir de cette station commence la portion eutrophiquée de la Semois. Les différences entre indices peuvent alors être expliquées : l'altération venant de Florenville a pu échapper à la chimie (prélèvement momentané) tout en étant enregistrée par les diatomées ; par ailleurs, c'est à partir de $S 7$ que, d'une part, l'eau est à nouveau bien pourvue en oxygène, ce qui peut expliquer l'apparition des Glossosomatidae, et que, d'autre part, on observe le développement de grands herbiers de macrophytes qui favorisent la diversification de la macrofaune: dans ces conditions, I'IBGN augmente.

Les stations S8 et S9 sont faiblement polluées. Le groupe indicateur 9 (plécoptères Perlidae) semble indiquer une amélioration. Dans certaines situations équivoques, AGENCE DE L'EAU (1995, p. 65) préconise de tester la robustesse de la note en calculant les indices avec et sans le premier groupe indicateur, et d'interpréter l'écart. Dans le cas présent, on obtient alors le groupe indicateur 7 moins sensible (Glossosomatidae) et des IBGN de 13 et 12 (pollution modérée à faible), au lieu de 15 et 14 . Cette proposition est judicieuse, car les 
plécoptères Perlidae sont réputés très exigeants en oxygène. Or, ces deux stations sont précisément les plus sursaturées (124 et $123 \%$ ), en raison de l'eutrophication. Dans ce cas, les Perlidae entraînent donc une surestimation de qualité.

Comme pour les diatomées, la station S10 est influencée négativement par les rejets de la station d'épuration de Bouillon (IBGN $=10$ ), bien que cela ait manifestement échappé à l'analyse chimique au moment du prélèvement. Les Perlidae et les Glossosomatidae disparaissent (manque probable d'oxygène dû aux eaux désaturées sortant de la station d'épuration) et le premier groupe indicateur est alors celui des Leptoceridae.

Enfin, les stations $\mathrm{S} 11$ et S12 sont proches de la S8 et sont faiblement polluées. Les Perlidae forment le groupe indicateur et quatre familles de trichoptères s'ajoutent (Goeridae, Odontoceridae, Philopotamidae, Rhyacophilidae).

Au niveau des affluents (tableau VI), la diversité ne dépasse pas 19 unités systématiques. Les stations $\mathrm{A} 1$ et $\mathrm{A} 3$ sont modérément polluées. En l'absence de Perlidae, les groupes indicateurs sont les Leuctridae pour $A 1$ et les Rhyacophilidae pour A3, groupes qui interviennent plus bas dans l'échelle de sensibilité. Nous n'expliquons pas cette absence, mais constatons que la chimie et les diatomées classent ces eaux en pollution faible.

Tableau VI : Peuplements d'invertébrés des affluents (résultats en nombres d'individus).

Table VI : Invertebrates of the tributaries of the river Semois (results in numbers of organisms).

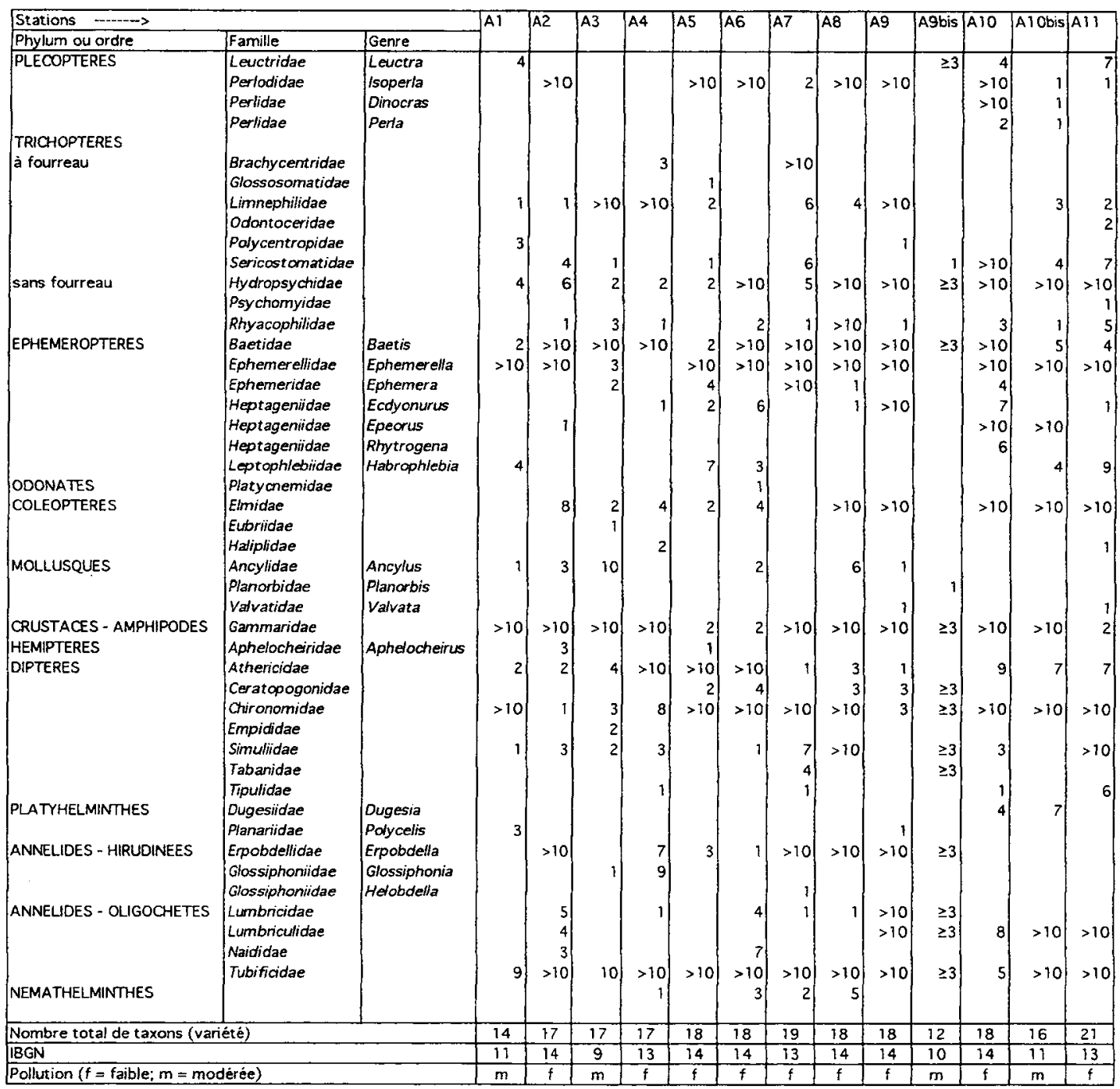


La station A2 est en pollution faible, en raison de la présence de Perlodidae. Elle est en pollution modérée en chimie et forte pour les diatomées. Si on applique le test de robustesse en écartant les Perlodidae (groupe indicateur 9 ), le groupe indicateur suivant (Sericostomatidae) est beaucoup plus bas (6) et l'indice de 11 correspond à une pollution modérée plus conforme aux autres analyses.

Rien n'indique que le ruisseau de Breuvanne (A4 : pollution faible) est de type calcaire, beaucoup plus minéralisé que les autres. Le groupe indicateur 8 (Brachycentridae) n'intervient que pour 3 individus : si on l'élimine, on tombe au groupe indicateur 3 (Limnephilidae) et à un IBGN de 8 (pollution forte). L'estimation correcte se trouve sans doute entre ces deux états, comme l'indiquent la chimie et les diatomées (pollution modérée).

Les stations A5 à A8 sont ici en pollution faible: les groupes sensibles (Perlodidae ou Brachycentridae) sont bien représentés et non isolés, les groupes indicateurs intermédiaires étant présents (Ephemeridae, Sericostomatidae, Leptophlebiidae, Heptageniidae). Rappelons que les diatomées montrent clairement que ces cours d'eau sont modérément eutrophiqués, ce qui ne gêne pas ces macro-invertébrés sensibles, pour autant que l'oxygène ne leur fasse pas défaut.

Les macro-invertébrés confirment la bonne qualité des stations $A 9, A 10$ et $A 11$, où les plécoptères sont bien représentés.

Les stations $\mathrm{A} 9 \mathrm{~b}$ is et $\mathrm{A} 10 \mathrm{bis}$ sont modérément polluées. On soulignera l'amélioration importante en A9bis depuis la mise en route de la station d'épuration de Bertrix, puisque des Leuctridae se sont réinstallés (ROSILLON, comm. pers.).

En conclusion, l'interprétation des IBGN, à partir des échantillons prélevés selon la méthode belge (DE PAUW et VANHOOREN, 1983), est délicate et demande une bonne connaissance de la faune ainsi qu'un recours constant au tableau de données, afin de moduler les valeurs indicielles. Le test de robustesse de la note IBGN, proposé par AGENCE DE L'EAU (1995), permet notamment de corriger les insuffisances de cette méthode, mais on ne peut évidemment le faire que si on dispose d'autres indices dont on connaît la fiabilité. Des problèmes surviennent surtout dans les cas difficiles de pollutions très variables ou d'intensité modérée, et dans les sites eutrophiqués.

Moyennant ces corrections, on distingue, pour la Semois, un cours supérieur très fortement pollué, un cours intermédiaire fortement pollué, un cours inférieur modérément à faiblement pollué. Les apports des affluents, même pollués, ne se marquent pas. Seul le rejet de la station d'épuration de Bouillon (S10) entraine une modification du peuplement d'invertébrés suffisante pour se traduire par une baisse de l'indice.

\section{SYNTHĖSE ET DISCUSSION (FIGURES 3 À 6)}

Sur la base des analyses chimiques, la Semois est subdivisée en trois secteurs :

- en S2 et $\mathrm{S} 3$ : pollution forte,

- de 54 à $\mathrm{S6}$ : pollution modérée,

- de S7 à S12 : pollution faible.

Nous laissons ici de côté la station S1, où la pollution chimique très forte (hydrocarbures) est probablement la cause d'une sous-estimation de la pollution organique (interférences).

La troisième zone correspond en fait à une zone eutrophiquée, riche en herbiers de renoncules (Ranunculus fluitans Lam.) et sursaturée en oxygène ; les matières organiques des rejets ont été minéralisées et les nutriments (nitrates et orthophosphates) sont mobilisés par ces végétaux, les teneurs dans l'eau étant faibles.

Au niveau de l'analyse diatomique, la Semois présente une succession d'états :

- en $S 1$ : pollution très forte,

- en $\$ 2$ et $\mathrm{S3}$ : pollution fortè,

- de S4 à S6 : pollution modérée (diminution de I'ID en aval de la Rulles), 
- en S7 : pollution forte (rejets de Florenville et de Martué),

- de S8 à S11: pollution modérée (diminution de I'ID en aval de Bouillon),

- en $\mathrm{S} 12$ : pollution faible.

Selon les diatomées, la zone la plus eutrophiquée est située en S3 et S4, en aval des rejets de nitrates et orthophosphates de la station d'épuration d'Arlon. Pour les phanérogames, l'eutrophication se marque surtout à partir de S7, mais il faut noter que le tronçon amont, jadis curé, rectifié et partiellement enroché, n'offre peut-être plus les mêmes potentialités d'accueil à des végétaux enracinés (VANDER BORGHT et SKA, 1989). En S8 et S9, on a un mélange d'espèces oligosaprobes et $\alpha$-mésosaprobes qui indique une irrégularité de la charge polluante, peut-être due aux rejets périodiques du barrage de la Vierre (turbinage). L'autoépuration arrive tout juste à faire passer la Semois en pollution faible, à la frontière franco-belge. L'eutrophication est aussi marquée dans la Vierre et ses affluents classés en pollution modérée.

Par l'analyse des macro-invertébrés, la Semois se divise en trois zones :

- de $\mathrm{S} 1$ à $\mathrm{S} 3$ : pollution très forte,

- de S4 à S6 : pollution forte,

- de S7 à S12 : pollution modérée à faible (diminution de l'IBGN en aval de Bouillon).

Dans la deuxième zone (pollution forte), on note un déficit de saturation en oxygène, un substrat rocailleux et peu de végétation phanérogamique. Dans ces conditions, les peuplements d'invertébrés sont peu diversifiés : les IBGN sont faibles par effet de substrat et ne reflètent pas l'amélioration de la chimie enregistrée par les diatomées (pollution modérée).

Cette description montre la complémentarité des trois types d'analyses : les analyses chimiques, pour quantifier les polluants à un moment donné ; les analyses diatomiques, pour décrire les effets des polluants sur les producteurs primaires et pour distinguer les différents états du milieu (eutrophisé, pollué, eutrophiqué) ; les analyses de macro-invertébrés, pour repérer des situations extrêmes (très bonnes ou très mauvaises) en examen de routine et des particularités locales liées aux substrats (rochers, graviers, sable, vase, végétaux, ...) et pour intégrer les dégradations de l'habitat (curages, enrochements ...).

La figure 5 établit un bilan complet pour deux stations clés de la Semois, en aval d'Arlon (S2) et à la frontière franco-belge, en aval de Bohan (S12). Les diagrammes ioniques sont ceux des types condrusien et famennien. La figure 6 illustre de la même façon, en station non polluée, les types ardennais mésotrophe (ruisseau des Fagnes, A3) et ardennais oligotrophe (Rulles en amont, A1).

En S2, la situation est critique : une pollution forte entraîne le développement de diatomées $\alpha$-mésosaprobes (en orange) et polysaprobes (en rouge). Les invertébrés sont limités à quelques chironomes et oligochètes, qui survivent dans un dépôt vaseux et organique. Il est clair que la station d'épuration d'Arlon n'est plus suffisante, vu le développement de la zone industrielle.

En S12, après un parcours de $160 \mathrm{~km}$, et malgré les nombreux apports non épurés, on arrive en pollution nulle à faible. Le peuplement de diatomées est dominé par les oligosaprobes (en vert), mais les $\beta$-mésosaprobes (en jaune) sont encore bien présentes. La faune benthique est la plus diversifiée parmi les 25 stations étudiées, mais elle est dominée par les groupes faunistiques résistants (en orange).

En $A 1$ et $A 3$, la qualité est bonne (dominance de diatomées oligosaprobes en vert), mais ces ruisseaux oligotrophes, souvent ombragés, de petite taille, avec un nombre limité de niches écologiques, ont une faune benthique peu abondante et peu diversifiée. L'IBGN sous-estime alors la qualité de ce type d'eau et la méthode ne convient pas. En outre, l'abondance des organismes considérés comme résistants par l'IBGN (en orange) surprend dans des eaux de bonne qualité. Nous pensons que le classement vertical des groupes indicateurs inférieurs ne correspond pas, dans tous les cas, à une résistance croissante à la pollution organique. C'est notamment vrai pour les Gammaridae (groupe 2 résistant), pourtant abondants dans toutes les eaux calcaires propres. 
SEMOIS (S2)
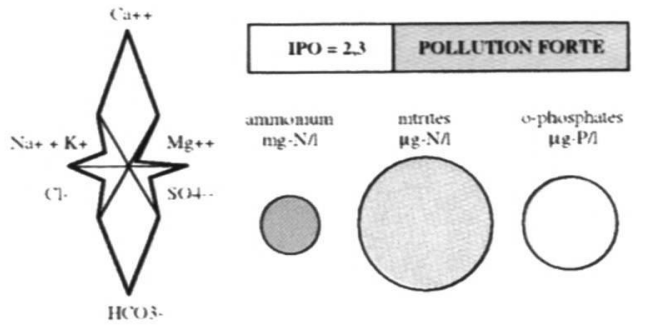

Dragrammes

wanlutes

$I_{0}^{1}$

meill
SEMOIS (SI2)

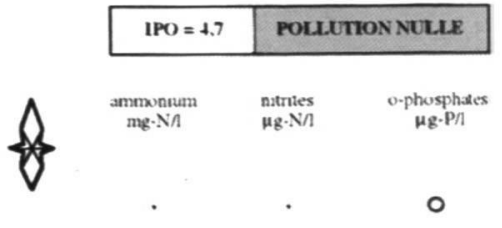

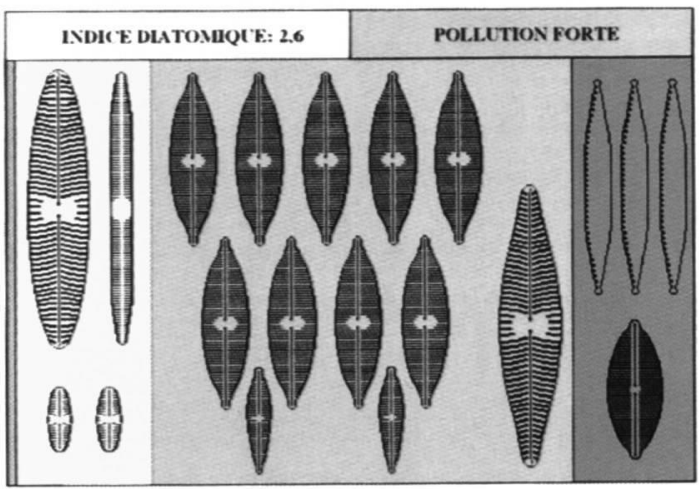
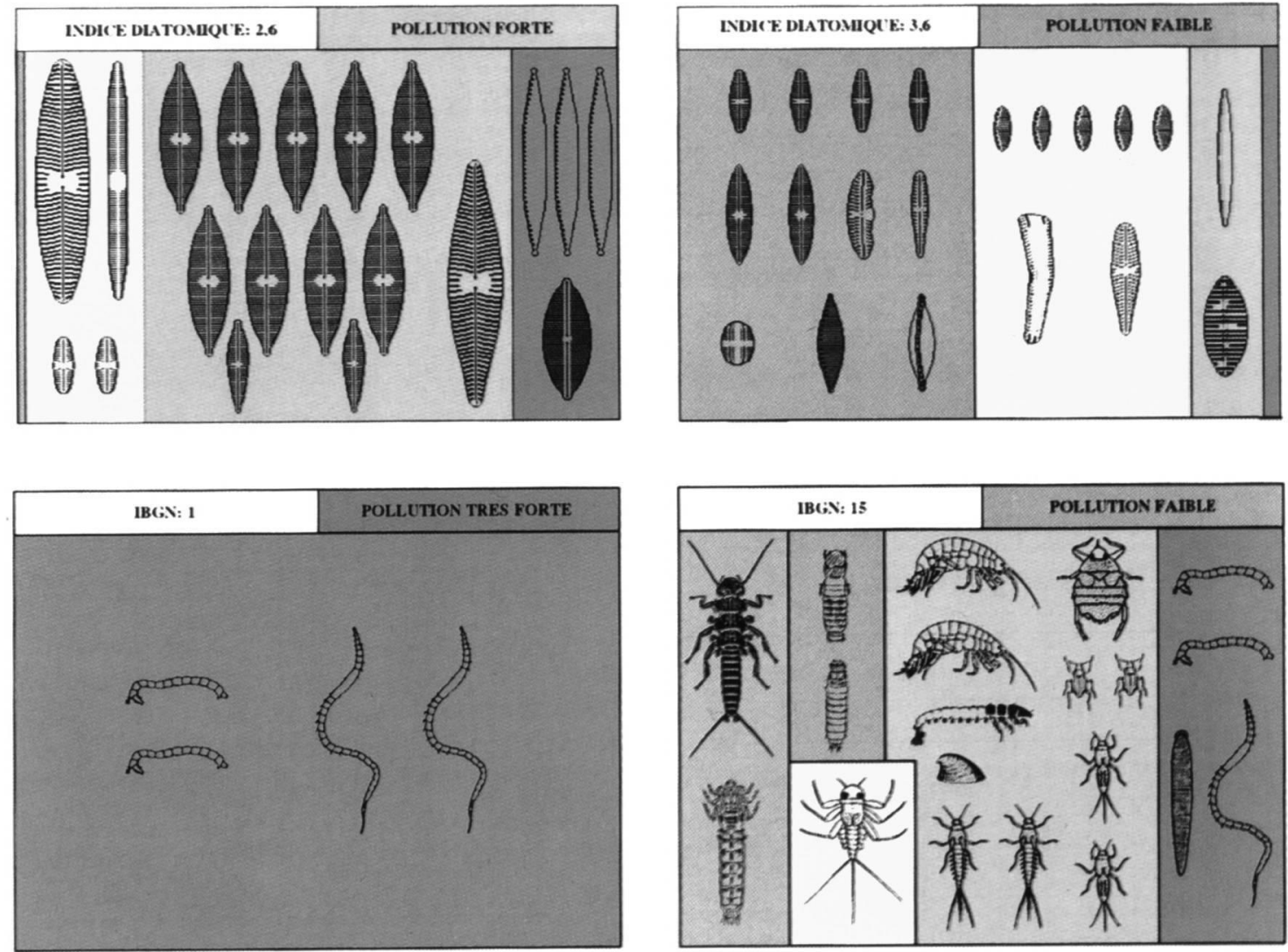

Figure 5 : Synthèse des trois analyses pour deux stations extrêmes de la Semois (S2 et S12). (Synthesis of the three analyses in two stations in the Semois, S2 and S12).

Légende des couleurs : voir tableau I et chapitre 2.3.

Légende des dessins de diatomées (nombres d'individus donnés comme repère) : en \$2: 1 Navicula lanceolata, 1 Fragilaria ulna, 2 Navicula minima (jaune), 9 N. gregaria, $1 \mathrm{~N}$. trivialis, 2 Gomphonema parvulum (orange), 3 Nitzschia palea, 1 Navicula accomoda (rouge); en $\$ 12$ : 4 Achnanthes minutissima, 2 Navicula cryptotenella, 1 Cymbella sinuata, 1 Gomphonema angustum, 1 Amphora pediculus, 1 Nitzschia fonticola, $1 \mathrm{~N}$. dissipata (vert), 5 N. inconspicua, 1 Rhoicosphenia abbreviata, 1 Gomphonema olivaceum (jaune), 1 Nitzschia paleacea, 1 Diatoma vulgare (orange).

Légende des invertébrés : en \$2 : Chironomidae et Oligochètes (rouge); en S12 : Perlidae, Odontoceridae (bleu), Goeridae, Lepidostomatidae (vert), Heptageniidae (jaune), Gammaridae, Hydropsychidae, Mollusques Ancylidae, Baetidae, Aphelocheiridae, Elmidae, Ephemerellidae (orange), Chironomidae, Achètes Erpobdellidae, Oligochètes (rouge). Dessins d'invertébrés d'après BERTRAND, 1954. 


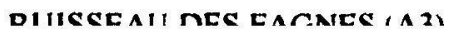

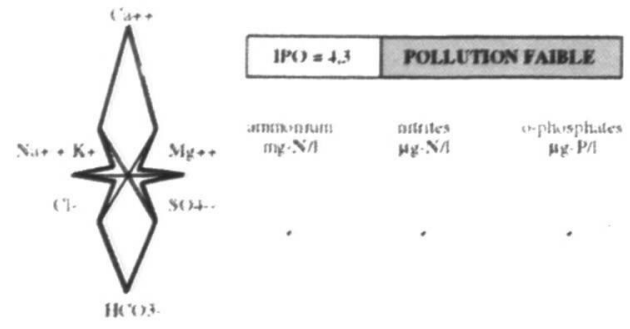

DIII CC, ^

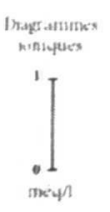

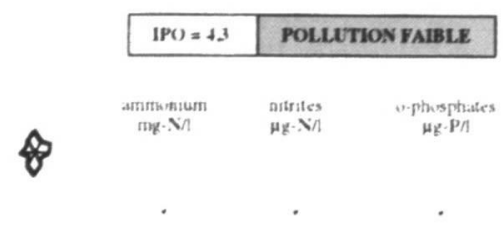
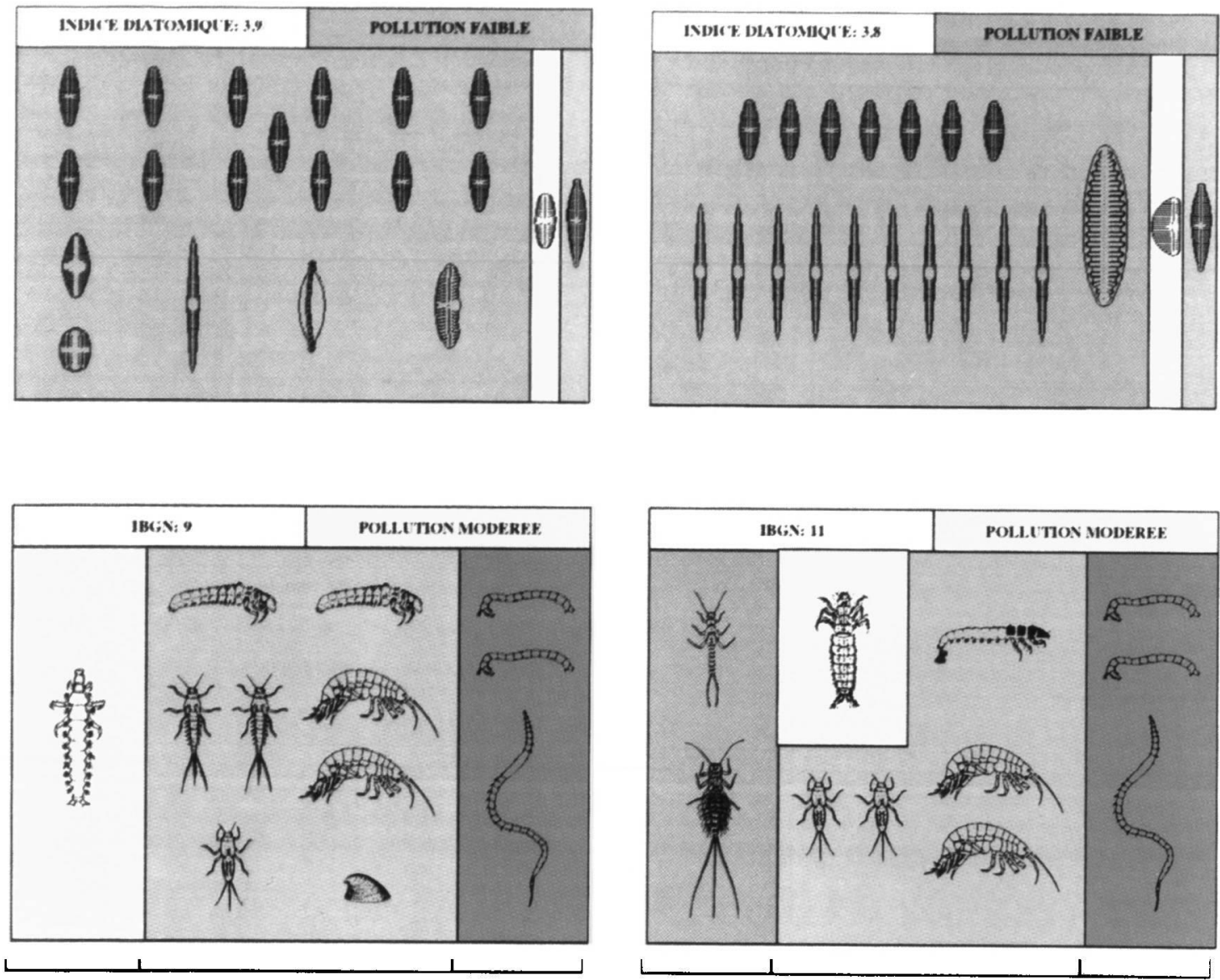

Figure 6 : Synthèse des trois analyses pour deux affluents de type ardennais mésotrophe et oligotrophe (A3 et A1).

(Synthesis of the three analyses in two tributaries of the Semois, A3 and A1).

Légende des couleurs : voir tableau I et chapitre 2.3.

Légende des dessins de diatomées (nombres d'individus donnés comme repère) : en $\mathrm{A3}$ : 13 Achnanthes minutissima, 1 Achnanthes laevis var. quadratarea, 1 Amphora pediculus, 1 Fragilaria capucina var. lanceolata, 1 Nitzschia dissipata, 1 Cymbella sinuata (vert), 1 Navicula minima (jaune), 1 Gomphonema parvulum (orange) ; en A1 : 7 Achnanthes minutissima, 10 Fragilaria capucina var. lanceolata, 1 Surirella roba (vert), 1 Cymbella minuta (jaune), 1 Gomphonema parvulum (orange).

Légende des invertébrés : en A3 : Rhyacophilidae (jaune), Limnephilidae, Baetidae, Ephemerellidae, Gammaridae, Mollusques Ancylidae (orange), Chironomidae et Oligochètes (rouge) ; en A1 : Leuctridae, Leptophlebiidae (vert), Polycentropidae (jaune), Hydropsychidae, Ephemerellidae, Gammaridae (orange), Chironomidae et Oligochètes (rouge). Dessins d'invertébrés d'après BERTRAND, 1954. 


\section{CONCLUSIONS}

Nous avons voulu présenter ici une étude très complète d'un bassin hydrographique. Pour ce faire, nous avons utilisé trois méthodes classiques : analyses chimique, diatomique et biocénotique.

Le bassin choisi, celui de la Semois belge, est original. Outre différents types d'eaux naturelles (oligotrophe, mésotrophe, eutrophe), on note, au niveau de la Semois, une diminution progressive de la minéralisation due au fait que les sources sont sur des roches calcaires du Jurassique et le cours inférieur sur des roches siliceuses du Dévonien inférieur. La relation entre la composition de l'eau et la géologie du bassin est très précise et permet, dans le cas présent, de confirmer la plus grande richesse en carbonates du Gedinnien par rapport aux autres assises du Dévonien inférieur.

Le bassin est touché par la pollution organique en maints endroits. Les paramètres qui la caractérisent (orthophosphates, ammonium, nitrites) sont très élevés en haute Semois et diminuent progressivement en aval. SYMOENS (1957) donne déjà un profil des orthophosphates, qui passent de $555 \mu \mathrm{g}-\mathrm{P} / \mathrm{l}$ à la source à $35 \mu \mathrm{g}-\mathrm{P} / \mathrm{l}$ après $170 \mathrm{~km}$. Au niveau des risques d'eutrophication, la situation de la Semois n'a pratiquement pas évolué depuis, malgré la mise en place de quelques stations d'épuration importantes. II faut y voir l'effet conjugué de l'absence de traitement tertiaire au niveau de ces stations et d'une augmentation des activités, notamment à Arlon (zone industrielle).

Ainsi que nous l'avons déjà montré (LECLERCQ, 1989), les peuplements de diatomées enregistrent fidèlement les caractéristiques chimiques des eaux, que ces caractéristiques soient naturelles (effet du calcium, eutrophisation) ou d'origine anthropique (pollution, eutrophication). Nous décrivons cinq niveaux typologiques naturels caractérisés par une minéralisation croissante (type ardennais oligotrophe, type ardennais à tendance mésotrophe, type ardennais mésotrophe, type famennien, type condrusien). Le choix des 25 stations permet donc d'établir une typologie de référence complète du bassin. II est alors possible d'évaluer correctement l'altération de ces cours d'eau. Les diatomées suivent étroitement le gradient de pollution et d'eutrophication, mettent en évidence l'effet des apports plus ou moins pollués des affluents et réagissent aux irrégularités des rejets par des peuplements composites.

L'analyse des macro-invertébrés ne permet pas de distinguer de types naturels : mis à part les mollusques, ces organismes sont indifférents au degré de minéralisation. Les valeurs indicielles suivent les mêmes tendances générales que les indices diatomiques (pollution très forte en amont, puis amélioration progressive), sans toutefois permettre de délimiter la zone eutrophiquée de la Semois, placée en pollution faible, ni de repérer l'effet des rejets polluants dans la moyenne et la basse Semois. Dans les affluents, aucune pollution forte n'est notée, mais plusieurs problèmes de surestimation ou de sous-estimation des IBGN sont interprétés à la lumière des analyses chimiques et diatomiques.

Au niveau de la méthodologie, le présent travail montre que les diatomées sont les plus aptes à enregistrer de manière fiable et fine les variations, même faibles, du milieu aqueux. Le prélèvement est rapide ( 5 minutes) et reproductible, l'identification à l'espèce est possible, le comptage est généralement aisé (1-3 heures). Le recours au spécialiste est, cependant, pratiquement obligatoire.

D'un point de vue fondamental, et aussi pour compléter utilement les rapports d'expertise, il est intéressant de pouvoir disposer, en plus, d'une analyse chimique, qui n'est qu'indicative puisque momentanée, mais qui permet de perfectionner progressivement la connaissance de l'auto-écologie des espèces de diatomées. L'idéal serait évidemment de pouvoir confronter l'analyse diatomique à une analyse chimique moyenne sur un laps de temps suffisant (3 semaines), mais le coût serait trop élevé.

Les peuplements d'invertébrés réagissent de façon moins précise et plus variable à la qualité de l'eau. Une augmentation ou une diminution des valeurs indicielles ne sont pas nécessairement corrélées à une amélioration ou à une dégradation. Deux cas de figure ont été 
rencontrés : des indices faibles qui sous-estiment la qualité de petites rivières oligotrophes, naturellement peu diversifiées ; des indices élevés qui surestiment la qualité en zones d'eutrophication, dans lesquelles des herbiers importants servent de biotopes à de nombreux invertébrés.

Si la méthode de l'indice biologique global normalisé (IBGN) constitue une nette amélioration par rapport aux indices biocénotiques antérieurs (IB, IQBG), elle reste encore trop sensible au facteur șubstrat. Sa mise en oeuvre isolément expose donc à des erreurs parfois importantes. Par ailleurs, ses auteurs (VERNEAUX et al.) ont voulu présenter une méthode simplifiée, accessible au plus grand nombre. C'est un objectif louable mais dangereux, car cette simplicité n'est qu'apparente. Par exemple, l'identification jusqu'à la famille n'est pas toujours évidente pour des non-spécialistes, surtout pour les stades larvaires les plus jeunes : des erreurs graves peuvent ainsi être commises. La simplification masque aussi une restriction évidente d'utilisation : on a vu ainsi la méthode appliquée abusivement pour des types d'eau pour lesquels elle n'était pas conçue (eaux dystrophes et oligotrophes, eaux lentiques, eaux saumâtres, canaux). La carte de qualité d'eau de la Belgique en est un exemple (VANHOOREN, 1986). II sera dès lors toujours utile de recourir à un spécialiste pour la vérification et l'interprétation des données. Notons enfin que le prélèvement selon la norme ( 8 biotopes) et le tri sont fastidieux et longs, ce qui entraîne une augmentation du coût par rapport aux analyses de diatomées.

Les responsables du contrat de rivière Semois et de l'épuration disposent maintenant d'un inventaire actualisé et très nuancé de l'état du bassin, à comparer avec les quelques données anciennes existantes. Plusieurs points critiques devraient attirer l'attention de ces gestionnaires: la zone industrielle d'Arlon sur la Semois, et les villages sur la Rulles et sur la Vierre supérieure, devraient être épurés prioritairement. L'eutrophication devrait être combattue par une épuration tertiaire au niveau des grandes stations existantes (Arlon, Neufchâteau, Bouillon).

On pourra alors voir la zone de pollution faible remonter progressivement vers l'amont, au profit de la faune piscicole.

Une analyse plus fine des invertébrés permettra ensuite de localiser les zones où des travaux malencontreux (curages, rectifications, destruction de berges, bétonnages, ...) ont appauvri les peuplements. Les gestionnaires pourront alors prendre des mesures de réhabilitation et penser les nouveaux travaux en fonction de la zoocénose, en restaurant des substrats favorables.

\section{BIBLIOGRAPHIE}

AFNOR, 1992. Détermination de l'indice biologique global normalisé (IBGN). Essais des eaux. Norme française T 90-350. Assoc. franç. de normalisation, Paris, $9 \mathrm{p}$.

AGENCE DE L'EAU, 1993. Étude bibliographique des méthodes biologiques d'évaluation de la qualité des eaux de surface continentales. Étude inter-agences $n^{\circ} 35$, I.D.E. Environnement. Synthèse bibliographique : 257 p. ; Guide méthodologique : $276 p$. + annexes ; Références bibliographiques : $41 \mathrm{p}$.

AGENCE DE L'EAU, 1995. Indice biologique global normalisé IBGN (NF T 90-350) : guide technique. Étude inter-agences $\left(n^{\circ}\right.$ spécial), Cabinet GAY-Environnement, $69 \mathrm{p}$.

BECKERS B., STEEGMANS R., 1979. De kwaliteit van de oppervlaktewateren in Limburg. Studiecentrum voor ecologie en bosbouw, Gent, rapport non publié.

BERTRAND H., 1954. Les insectes aquatiques d'Europe. Encyclopédie entomologique, Lechevalier, Paris, Vol. 1:556 p. ; vol. 2 : 547 p.

DE PAUW N., VANHOOREN G., 1983. Method for biological quality assessment of watercourses in Belgium. Hydrobiol., 100, 153-168. 
DESCY J.P., 1978. Étude préliminaire des peuplements de diatomées benthiques de la HauteSemois en vue d'une évaluation de la qualité biologique des eaux. F.U.L., Notes de recherche 15, Étude des eaux superficielles du bassin de la Meuse, 8-24.

DESCY J.P., EMPAIN A., LAMBINON J., 1982. Un inventaire de la qualité du bassin wallon de la Meuse (1976-1980). Trib. CEBEDEAU, 463-464, 257-278.

DUVIGNEAUD P., 1984. La synthèse écologique. Populations, communautés, écosystèmes, biosphère, noosphère. Doin (ed.), Paris, 2ème édition, $380 \mathrm{p}$.

FABRI R., LECLERCQ L., 1984. Étude écologique des rivières du nord du massif ardennais (Belgique) : flore et végétation de diatomées et physico-chimie des eaux. Robertville, Stat. scient. Hautes-Fagnes, $1: 379$ p., 33 pl. h.t. ; $2: 5+329$ p. ; $3: 5+201$ p.

HUSTEDT F., 1927-1966. Die Kieselalgen. In L. Rabenhorst's Kryptogamen-Flora von Deutschland, Österreich und der Schweiz. Leipzig, Akad. Verlagsges., 1 (1927-30): 12 + 920 p. ; 2 (1931-59) : 12 + 845 p. ; 3 (1961-66) : 816 p.

KOLKWITZ R., MARSSON M., 1908. Ökologie der pflanzlichen Saprobien. Ber. deutsch. bot. Ges., 26a, 505-519.

KRAMMER K., 1992. Pinnularia. Eine Monographie der europäischen Taxa. Bibliotheca Diatomologica, 26, $353 \mathrm{p}$.

KRAMMER K., LANGE-BERTALOT H., 1986-1991. Süßwasserflora von Mitteleuropa. Bacillariophyceae. 1. Naviculaceae : 876 p. (1986) ; 2. Bacillariaceae, Epithemiaceae, Surirellaceae : 596 p. (1988) ; 3. Centrales, Fragilariaceae, Eunotiaceae : 576 p. (1991) ; 4. Achnanthaceae : 437 p. (1991). G. Fischer Verlag, Stuttgart.

KUFFERATH J., 1951. Représentation graphique et classification chimique rationnelle en types des eaux naturelles. Bull. Inst. roy. Sc. natur. Belg., 27 (43), 8 p., 1 pl.'h.t. ; 27 (44), 6 p. ; 27 (45), $8 \mathrm{p}$.

LECLERCQ L., 1984. Composition chimique des eaux des rivières oligotrophes de haute Ardenne (Belgique) et ses variations saisonnières. Physio-Géo, 9, 139-152.

LECLERCQ L., 1988. Recherche sur l'épuration par marais reconstitué en vraie grandeur et application à la gestion du réseau hydrographique du Viroin et de l'Hermeton. Rapport 4 : Méthodologie pour la détermination d'objectifs de qualité dans un parc naturel. Synthèse des analyses physico-chimiques de mars à décembre 1987 dans l'Hermeton et la Joncquière. 22 p., 9 fig. et 15 tab. h.t., Centre Marie-Victorin, Vierves-sur-Viroin (ed.).

LECLERCQ L., 1989. Fiabilité de l'estimation du niveau de pollution organique des eaux courantes par les diatomées et efficacité de leur pouvoir intégrateur. Cryptogamie Algologie, 10 (2), 87-100.

LECLERCQ L., 1991. Intérêt et limites des méthodes d'estimation de la qualité des eaux courantes. Syllabus de cours. FUL, Arlon, 56 p.

LECLERCQ L., MAQUET B., 1987. Deux nouveaux indices chimique et diatomique de qualité d'eau courante. Application au Samson et à ses affluents (bassin de la Meuse belge). Comparaison avec d'autres indices chimiques, biocénotiques et diatomiques. Inst. roy. Sc. nat. Belg., Document de travail 38, 113 p.

LECLERCQ L., VANDEVENNE L., 1987. Impact d'un rejet d'eau chargée en sel et d'une pollution organique sur les peuplements de diatomées de la Gander (Grand-Duché de Luxembourg). Cahiers de Biol. Mar., 28(2), 311-318.

PIERRE J.F., 1977. Algues et mortalité piscicole en Semois. Technique de l'Eau et de l'Assainissement, 368-369, 19-31.

PRATI L., PAVANELLO R., PESARIN F., 1971. Assessment of surface water quality by a single index of pollution. Water Research, 5, 741-751. 
ROUND F.E., 1993. A review and methods for the use of epilithic diatoms for detecting and monitoring changes in river water quality. HMSO Books, London, $65 \mathrm{p}$.

SLADECEK V., 1973. System of water quality from the biological point of view. Arch. Hydrobiol. Beih. Ergebn. Limnol., 7, 4 + 218 p.

SYMOENS J.J., 1957. Les eaux douces de l'Ardenne et des régions voisines : les milieux et leur végétation algale. Bull. Soc. roy. Bot. Belg., 89, 111-314.

VANDER BORGHT P., SKA B., 1989. Eutrophisation et qualité des eaux de la Semois. Trib. Eau, 42, 7-11.

VANDER BORGHT P., ROSILLON F., 1994. Le contrat de rivière Semois. Environnement et Société, 13, 29-31.

VANHOOREN G., 1986. Carte de la qualité biologique des cours d'eau de la Belgique. Bilan pour 1985. Min. Santé Publique et Famille, IHE, 24 p., 1 carte h.t.

VERNEAUX J., TUFFERY G., 1967. Une méthode zoologique pratique de détermination de la qualité biologique des eaux courantes. Indices biotiques. Ann. Sc. Univ. Besançon, 3, 79-89.

VERNIERS G., MICHA J.C., 1982. Le rôle de l'hydrobiologiste dans l'évaluation de l'impact des activités humaines sur les milieux aquatiques. Trib. CEBEDEAU, 460, 117-131. 